\title{
Identity and divergence of protein domain architectures after the Yeast Whole Genome Duplication event
}

L. Grassi ${ }^{2, \ddagger}$, A. L. Sellerio ${ }^{1, \ddagger}$, D. Corà ${ }^{2,3}$, B. Bassetti ${ }^{1,4}$, M. Caselle ${ }^{2,3}$, M. Cosentino Lagomarsino ${ }^{1,4 *}$

${ }^{1}$ Università degli Studi di Milano, Dip. Fisica. Via Celoria 16, 20133 Milano, Italy.

${ }^{2}$ Università degli Studi di Torino, Dip. Fisica Teorica, Via Giuria 1, 10125 Torino, Italy.

${ }^{3}$ I.N.F.N. Torino, Via Giuria 1, 10125 Torino, Italy

${ }^{4}$ I.N.F.N. Milano, Via Celoria 16, 20133 Milano, Italy

* : corresponding author, email: Marco.Cosentino-Lagomarsino@unimi.it, Tel. +3902 50317477 ;

$\ddagger$ : equal contribution. 


\begin{abstract}
Analyzing the properties of duplicate genes during evolution is useful to understand the development of new cell functions. The yeast $S$. cerevisiae is a useful testing ground for this problem, because its duplicated genes with different evolutionary birth and destiny are well distinguishable. In particular, there is a clear detection for the occurrence of a Whole Genome Duplication (WGD) event in S. cerevisiae, and the genes derived from this event ("WGD paralogs") are known. We studied WGD and non-WGD duplicates by two parallel analysis based on structural protein domains and on Gene Ontology annotation scheme respectively. The results show that while a large number of "duplicable" structural domains is shared in local and global duplications, WGD and non-WGD paralogs tend to have different functions. The reason for this is the existence of WGD and non-WGD specific domains with largely different functions. In agreement with the recent findings of Wapinski and collaborators (Nature 449, 2007), WGD paralogs often perform "core" cell functions, such as translation and DNA replication, while local duplications associate with "peripheral" functions such as response to stress. Our results also support the fact that domain architectures are a reliable tool to detect homology, as the domains of duplicates are largely invariant with date and nature of the duplication, while their sequences and also their functions might migrate.
\end{abstract}




\section{Introduction}

Genomes possess a high degree of redundancy in the information they encode for [1, 2, 3, 4, 5]. Considering protein-coding genes, there is strong evidence [6, 7] that this redundancy has arisen from gene duplication events. Such duplications can involve individual genes, genomic segments or whole genomes. The yeast $S$. cerevisiae has arisen from an ancient whole-genome duplication [8].

The study of gene duplications is useful for understanding the evolution of proteins. Proteins descending from a common ancestor (homologs) are usually identified by sequence alignment methods. However, such methods typically have two main hindrances: (i) not taking into account directly the protein folding, which persists on longer evolutionary time scales than protein sequence, and (ii) being computationally intensive. On the other hand, structure and function of proteins can be described on a coarser scale, considering the protein domains, modular substructures that are defined by folding [9], compact structure [10, function and evolution [11]. Several authors [12, 13, 14, proved the usefulness of structural domain assignments in identifying homology. This implies that the duplicates tend to maintain their structures. This observation raises two interesting questions. The first one is how reliable the structural homology assignment is and whether it provides insight about the evolution of duplicates. The second question is whether it is possible to use domain architecture information combined to functional annotation for the characterization of duplicates from global and local duplications at different dates.

We addressed the first question by implementing an algorithm for detecting homology via structural domain assignments and comparing the results with the ones obtained by sequence alignment methods. More specifically, the description of genes at the protein domain level requires: (i) the construction of a protein domain architecture database, containing a description of each protein, in term of the domains that form it; (ii) the implementation of homology criteria between the entries of the database. This method is limited by our partial knowledge of protein domains, so that the architecture data suffer from incomplete coverage. Furthermore, the choice of an homology criterion implies a trade-off between error tolerance and the rate of false-positive homologs. We studied the evolution of WGD paralogs by comparing the structural domain architecture also considering their K. waltii orthologs.

The second question arises from the fact that gene duplications drive evolutionary innovation, by providing raw material to develop new functions. In particular it is interesting to understand how the whole-genome duplication event reshape the genome in a distinct way from local duplications and how this is reflected by the domain structure of duplicates. We used our method for evaluating differences between WGD paralogs and non-WGD paralogs, 
and performed a parallel comparison using a Gene Ontology enrichment analysis. Both analyses converge on the conclusion that whole-genome and local duplicates tend to be functionally different. Generally, core functions are enriched for WGD paralogs, while peripheral functions are enriched for non-WGD paralogs. Since domain structures of duplicates are essentially maintained, this dichotomy can be created by two main factors, for both of which we find evidence. The first one is the difference between domains that are preferentially duplicated in global and in local duplications. The second one is the migration of subcellular localization and specificity for given biological pathways.

\section{Results}

\section{Homology assignment by domain characterization}

The superfamily domain coverage spans one third of the genomes we examined. According to the SUPERFAMILY database, v. 1.69 [15], for S. cerevisiae there is a total of 6702 sequences, $3346(50 \%)$ of which with at least one assignment. The coverage is approximately $34 \%$ of total sequence, and $85 \%$ of domains are produced by duplication. The figures for K. waltii are similar: $2932(56 \%)$ sequences on 5214 were given at least one assignment, representing the $36 \%$ of total sequence covered; $84 \%$ of domains are produced by duplication.

In order to study homology from the structural domain viewpoint, we implemented three homology criteria based on domain architectures [14. Criterion A defines two proteins as homologs if their domains architectures coincide (i.e. they contain the same domains in the same order). Criterion B allows for multiple repetitions of the same domains. The biological hypothesis behind this criterion is that, after duplication, changes may occur to the architecture of the proteins, by mechanisms such as internal duplication (e.g. by unequal crossing over), generating architectures containing multiple repetitions of one or more ancestral domains. Finally, two proteins are identified as homologs by criterion $\mathbf{C}$ if their architectures are equal, or if one of them is an approximate repetition of the other (see Methods). Biologically, this choice is motivated by the fact that it allows to recognize the simplest events of recombination, and is more fault-tolerant to differences in structural assignments generated by lack of knowledge, i.e. gaps in domain architectures.

We compared homology classes generated by the three criteria with those defined by sequence alignment methods. This test was divided in two different steps.

First, we evaluated the fraction of homology relationships identified for the WGD (by Kellis et al [8]) and by general sequence alignment methods (Ensembl-Compara [16]) that are also identified by criteria $\mathbf{A}, \mathbf{B}$, and $\mathbf{C}$. 
The results of this analysis are shown in Table 1. These results confirm the efficiency of domain-based classifications in detecting evolutionary relatedness among proteins (as observed in [17]). Specifically, they indicate that even the most stringent homology criterion $\mathbf{A}$, is able to find the majority of triplets (72\%), pairs (67\%) and Ensembl Compara homology classes (64\%). The other criteria perform better; in particular, criterion $\mathbf{C}$ retrieves more than $90 \%$ of the information in blocks of conserved syntheny. The results indicate that this method detects every type of homology (orthology and paralogy, both general and WGD).

Secondly, we quantified the fraction of paralogs not recovered by Ensembl, for each paralogy class defined by the three homology criteria (figure 11). All three criteria define a significant fraction of classes that are not recovered by Ensembl. Notice that criteria $\mathbf{A}$ and $\mathbf{B}$ follow qualitatively similar trends and produce a small fraction of partially covered classes, while criterion $\mathbf{C}$ has a larger number of partially covered classes, essentially due to the fact that classes produced with this criterion are very large. Criterion $\mathbf{B}$ is the most efficient of the three criteria in returning Ensembl Compara paralogy relations. Figure 1 shows the limitations of both criterion $\mathbf{A}$ and C. The former, being more restrictive, builds small homology classes and consequently the probability that a whole class is not recognized by Ensembl is higher. The latter builds wide homology classes associating far away homologs. The consequence is that the classes built with criterion C will almost certainly contain some Ensembl homologs, as shown by the small number of classes that are not recovered. On the other hand, the same classes rarely contain Ensembl homologs only and consequently are rarely completely covered.

\section{Domain architecture evolution in WGD and non-WGD dupli- cates}

Duplicate gene pairs must undergo an altered selective regime that leads to an asymmetry emerging at different levels, for example as an increase in the rate of protein sequence evolution. Furthermore, genes at fixation may evolve in different ways, depending on the divergence process and the nature of the duplication [18. Among the possibilities, there is a process by which one copy maintains the original function, and thus is constrained by selection, leaving the other one free to evolve, as originally hypothesized by Ohno [19] and supported by evidence in yeast [5, 20, 21]. However, theoretical and experimental work has argued that both duplicates can evolve independently at the same rate [22, 23]. We considered the question of testing the consequences of these processes at the domain level.

We followed the evolution of WGD duplicates through their domain architectures, i.e. the ordered sequence of domains forming the proteins. The length of an architecture is the total number of domains and gaps that form 
it. There are three main processes that affect architecture evolution: (i) growth by internal duplication, (ii) sequence divergence leading to structural changes in domains and (iii) domain insertions. In order to quantify globally the changes in protein architectures, we introduced two scoring methods that define a quantitative notion of relatedness between architectures. The first, called "domain score" is the number of domain types shared by two proteins over the sum of all domains of both proteins taken only once. The second "architecture score" measures the longest exactly matching sequence of domains between two architectures, divided by the mean length of the two architectures (see Methods).

To test for asymmetry, we compared for each WGD triplet the two $S$. cerevisiae WGD paralogs with the respective K. waltii ortholog, detecting the best- and worst- matching paralog. This was done using the domain score and the architecture score between both paralogs and their $K$. waltii ortholog. Table 2 shows the fraction (F2) of WGD triplets in which both $S$. cerevisiae duplicates have identical domain (or architecture) scores to their WGD ortholog in $K$. waltii. Furthermore we called F1 the fraction of triplets in which only one of the two $S$. cerevisiae paralogs has domain (or architecture) score one with the corresponding $K$. waltii ortholog. Comparing proteins with the architecture score we detect $65 \%$ of $\mathrm{F} 2$ triplets and $16 \%$ of $\mathrm{F} 1$ triplets, while by using the less restrictive domain score we detect $80 \%$ of $\mathrm{F} 2$ triplets and $11 \%$ of $\mathrm{F} 1$ triplets 2. This indicates that some duplicate proteins tend to evolve without changing their domain composition but rather by changing their order. We compared these results with a null model that performed random shuffling of the empirical values of the scores between the fixed sets of ortholog pairs (thus erasing the correlation between scores of the same triplet, see Methods). Interestingly, the randomized histograms show specular trends for the distribution of the scores for the two graphs that are more enhanced than for the empirical case. These data indicate that the difference between rates of "divergence" of protein architectures of WGD paralogs compared to their ortholog in K. waltii is larger in randomized instances. Consequently, the domain architectures of WGD duplicates are typically more balanced than expected from the null model, rather than more asymmetric.

We extended the analysis of paralog divergence to non-WGD paralogs, taking into account the duplication date reported by Wapinski et al. 24. Measuring the average domain and architecture scores as a function of duplication age, and their standard deviations on the age sets, we find that the domain score is roughly constant and very close to one (figure 21), indicating that even ancient paralogs maintain similar domain composition. The more stringent architecture score shows a similar trend, with a more marked decrease for pre-WGD paralogs. Note that the proximity to one of the domain score implies that the same score for single-copy orthologs cannot be much higher, and thus that the observed accelerated evolution of 
paralogs [20] should not be seen at the domain level. In order to test this directly, we have considered the distribution of domain and architecture score for single-copy $S$. cerevisiae genes versus their $K$. waltii orthologs and we have compared this result with domain and architecture score of doublecopy S. cerevisiae genes (WGD duplicates) versus their $K$. waltii orthologs. The two histograms perfectly overlap for both architecture and domain score (figure S1).

\section{Functional divergence and duplication age}

In order to gain more insight into the divergence of duplicates at the domain level, we evaluated how the same duplicate proteins tend to diverge in their function. Specifically, we calculated the Gene Ontology (GO) term similarity between paralogs for each of the GO branches ("molecular function", "biological process" and "cellular component") by using the GOSim package 25. The results, shown in figure 3, indicate that for all the three GO branches, recent duplicates tend to be more similar than older ones. Indeed, average GO term similarity values tend to decrease as the duplication time increases. On the other hand, the mean GO term similarity of duplicates in all duplication date group never reach values lower than one half, indicating that also ancient pre-WGD duplicates tend to maintain some functional overlap. The curve of GO similarity versus duplication age reaches lower values for the "biological process" and "cellular component" branches. This indicates that duplicates are more likely to diversify the biological process they participate into and the cellular compartment to which they belong rather than their molecular function. Secondly, they do so at domain score nearly fixed to a value close to one, indicating that on average the function of duplicates migrates within the same fold structure, presumably by sequence mutations or recombinations maintaining the same structural domains [26].

The same trends are also visible from the histograms of GOsim and domain-based similarity scores of all duplicate pairs (figure 4). The pairs of duplicates having high domain-based similarity is consistently higher in number than those with high GO similarity, but this trend is weaker for the "molecular function" taxonomy. In order to gather more direct evidence of this general domain and functional conservation under strong sequence evolution, we also compared these figures with the normalized histogram of the (protein) sequence identity (\%id/100) between pairs of duplicates from Smith-Waterman pairwise alignments, performed by using EMBOSS Water [27] (figure 44). The latter distribution has the lowest peak at one and the highest value at low scores, confirming that strong migration in protein sequence accompanies stability of domains and functions.

In order to exclude biases of computational nature that could influence the results, we repeated the analyses with different conditions. Firstly, not all proteins $S$. cerevisiae are covered entirely by domains, but some have 
gaps. Excluding from the analysis proteins with gaps should confirm that the functional migration of paralogs is not attributable to unknown domains. Supplementary figure S4 shows that this is indeed the case. Secondly, Gene Ontology annotations inferred from computational evidence could generate false positives in GO similarity, especially in the case of recent duplicates with significant sequence similarity. To circumvent this possibility, we restricted the analysis to manually curated genes. This filter reduces significantly our dataset, especially in the case of non-WGD duplicates. For this reason, we grouped non-WGD paralogs in two pre- and post-WGD sets. This gave sufficient statistics to retrieve the same trends of figure 3 for the "biological process" and "molecular function" GO branches (supplementary figure $\mathbf{S 3}$ ), but not in the case of the "cellular component" GO branch, where the data are insufficient.

\section{Functional connotation of WGD and non-WGD paralogs}

Next, we focused on the difference in function between local and global duplicates. Whole-genome and local duplications are different biological processes, and the analysis of WGD and non-WGD paralogs can help understanding the biological constraints laying behind the different processes leading to long-term persistence of duplicated pairs in the two cases [24, 28, 29]. In particular, different works proved that WGD and non-WGD duplicates are enriched for different functional classes of genes. Thus, we set out to quantify with our methods how the effects of the WGD on the genome are qualitatively different from those brought by local duplications.

\section{Domain-based analysis}

Functional assignment of domains can be used for evaluating the evolutionary destiny of duplicates. We considered two functional classifications for domains given in the SCOP database [6, 7. We then proceeded to evaluate the trends in domain duplications, regardless from the specific protein they were duplicated with. We assigned domains to a set $\mathcal{O}$ if they were duplicated in at least one WGD paralog, and a set $\mathcal{P}$ if they appeared in at least one local duplication (see Methods). We considered paralogs the genes that are recognized by homology criterion $\mathbf{B}$ and do not belong to set $\mathcal{O}$. First, we found that the intersection of these two sets, in the universe of all SUPERFAMILY domains, is larger $\left(\mathrm{P}\right.$-value $<10^{-28}$ ) than expected from a hypergeometric null model (figure 5). Thus, there is a dominant common set of domains that is prone to be duplicated, regardless of the local or global duplication mode.

On the other hand, the observed distribution of the fraction of WGD versus non-WGD duplicate proteins where each domain topology is found is very uneven (supplementary figure S5). This trend indicates the existence of 
two populations of domain topologies: those that are duplicated only outside the WGD, and those that appear in both kinds of duplications, but have a bias towards being found in the WGD only. Consequently, we analyzed the sets $\mathcal{O} \backslash \mathcal{P}$, the domains only found in WGD duplicates, and $\mathcal{P} \backslash \mathcal{O}$, the domains only found in non-WGD duplicates, for functional enrichment. For the finer categories of the SCOP functional classification we found a few cases where the enrichment was biased in two opposite ways in the two sets, i.e. categories having a positive $Z$-score for WGD domains, and a negative $Z$-score for non-WGD domains.

The categories that show a bias for WGD-specific domains (belonging to $\mathcal{O} \backslash \mathcal{P}$ ) correspond to functions that are growth-related (ribosomes, translation), involved in regulation of gene transcription and degradation (transcription factors, proteases), primary metabolism (coenzymes) or cell adhesion. On the other hand, a positive bias for locally duplicated domains (belonging to $\mathcal{P} \backslash \mathcal{O}$ ) was found in functional categories related to transport, post-transcriptional regulatory processes and secondary metabolism. Surprisingly, we found that the category DNA repair and replication tends to be enriched among domains duplicated locally rather than globally. Weaker signals for the same trend were found for RNA processing and modification, chromatin structure and dynamics, toxins and defense enzymes.

\section{Gene Ontology analysis}

In parallel, we performed a more standard functional characterization based on Gene Ontology analysis on the proteins, along the lines of previous studies [28, 29]. We considered the disjoint sets of WGD and non-WGD paralogs. For each set we extracted the over-represented GO terms, and we compared them looking for the terms shared between WGD and non WGD-paralogs or specifically connected to a group (over-represented in a group and not significantly present in the other). WGD and non-WGD paralogs are enriched in different GO terms. We performed the same analysis also on randomized sets. Two randomly assorted sets tend to share more over-represented GO terms than WGD paralogs and non-WGD paralogs. These results are inverted considering the terms specific for each group: differently from the random assorted groups, WGD paralogs and non-WGD paralogs have many exclusive genes (see Supplementary Results), indicating that WGD and nonWGD paralogs carry out different functions.

In accordance with the domain-based analysis and with the previous hierarchical analysis derived from expression profiles and functional annotations [24, 28, we find that WGD paralogs are enriched for genes involved in "fundamental" processes such as for example, ribosomes and translation, regulation of cell cycle, regulation of developmental processes, sporulation, NADP metabolic process. On the other side the non-WGD paralogs are enriched for genes involved in "peripheral" processes such as transport, amino 
acid transmembrane transport, cellular wall, vitamin metabolism.

Finally, a recent study by Guan and coworkers 28] found that WGD duplicates are more likely to share interaction partners and biological functions than non-WGD duplicates. To confirm the latter result, we analyzed the distribution of the GO similarity normalized histograms for all the pairs of the two disjoint sets. Indeed, WGD paralogs result slightly more similar than non-WGD paralogs for all the three GO branches (supplementary figure (S2). On the other hand, comparing with figure 3, one notices that pre-WGD paralogs are less similar at the functional level, so that this signal might come at least in part from the functional difference of ancient non-WGD paralogs.

\section{Discussion}

Homology among distant paralogs and orthologs proteins is a difficult task because of sequence divergence. But it is well known that the structure of a protein is more conserved than its sequence. To score distant relationships among yeast and $K$. waltii proteins we used SCOP superfamilies domain assignments. This choice has three main reasons. First, these domains contain three-dimensional structural information, and are not solely based on sequence similarity, so that they can be considered, at least to a certain extent, "independent" from sequence alignments. Second, compared to the higher classification into "folds", they are defined to guarantee monophyly, excluding convergent evolution. Evolutionary information on domains is intrinsic of the classification scheme of the SCOP database, which is the basis for the hidden Markov models of the SUPERFAMILY database. Third, this choice was taken in previous studies [17, 30, which give a term of comparison.

The criteria and scores we used assume that two proteins derived from the same common ancestor if they have the same domain architecture, or a series of domains from the same protein families. This method allowed us to compare the more distant structural homology relationships with those obtained by sequence comparisons alone, and it also provided us with simple means to study the evolution of protein function from the structural viewpoint, at the genome-wide level. Naturally, the hidden Markov model assignment of domains depends on the scoring parameters. We limited our analysis to the criteria used by the SUPERFAMILY database [14, 17, 30]. A thorough analysis of the role of these parameters is presented in ref. [7].

Domain architecture and homology. Despite the sparse coverage of structural domains, it seems evident from our results that even elementary domain based homology criteria can recover most of the information obtained through sequence alignments techniques. Indeed, the criteria we 
defined are able to capture a large fraction of Ensembl-Compara homology classes, and behave similarly for local duplications or the WGD. On the other hand, the opposite is not true. Several domain-based homology relationships are not found by sequence alignment methods. We quantified this by measuring the fraction of domain-based homology classes not containing Ensembl-Compara classes. Criteria $\mathbf{A}$ and $\mathbf{B}$ have a similar percentage of homologs not detected in Ensembl, while criterion $\mathbf{C}$, follows a different trend. This last criterion is the only one that allows for insertion of external domains after duplication, which is an event that has been observed and can be expected from our knowledge of the evolutionary dynamics of proteins [31, 32, 33].

On the other hand, the different behavior of criterion $\mathbf{C}$ could suggest a lower reliability compared to the other ones. It is important to stress that the architecture comparison methods implemented in this paper can show false-positive matches. In other words, the less restrictive the criterion is, the higher is the possibility to incorrectly identify evolutionarily unrelated genes as homologs.

Overall, while some instances could represent false positives, we believe it is natural to expect that some others represent distant relationships that are not detected as paralogs by sequence alignment methods, but are recognized by domain-based methods. Our tools do not allow to quantify these false positives directly. However, we have accessed some other observables that go in favor of the reliability of domain-based criteria. Firstly the mean domain scores and, to a certain extent, the mean architecture scores of duplicates are very close to one, and remain invariant with duplication age (figures 22). This indicates that even ancient paralogs tend to have very similar domain composition. The slight drop of the architecture score for ancient pre-WGD duplicates suggests that even if paralogs tend to maintain their domain composition, the domain order or the number of repetitions may vary. Secondly, WGD paralogs do not show any peculiarities at the domain level compared to local duplicates and single-copy orthologs. On the contrary, there exists a significantly large set of "duplicable" domains, shared by the two duplication modes. This is in contrast with the markedly decreasing trend followed by the GO similarity score between paralogs as a function of duplication age, indicating that domains remain stable as protein function and sequence drift. Together, these data show that both the domains composing a protein, and the domain architectures are rather stable and independent from the specific evolutionary history, which goes in favor of homology criteria based on this aspect.

Thus, the above evidence goes in favor of using structural domains as a simple and computationally effective tool to discover gene duplications. At the same time, it points to some limitations of these methods. The most important of these is that currently no tool is available to quantify the failure rate of domain-based methods in detecting gene duplications. In 
other words, it would be important to estimate precisely which fraction of paralogs detected by domain-based methods and not by sequence alignment are really significant. For example, one cannot exclude that genes gained by horizontal transfer give rise to proteins with the same domain structure as some other proteins in the genome [34, or that the partial coverage of domain databases does not enable to resolve distinct architectures. However, an exact quantification of these processes is lacking.

Domain structure and function of duplicate proteins. A second and more biological question is to use domain architectures to understand gene duplication, and in particular the differences between local duplications and the WGD. To approach this question, we compared the results of our domain similarity scores with a functional evaluation at the level of both domains and genes.

Following duplications, proteins show divergence in their domain architectures. Our scoring criteria quantify the rate of divergence of architectures. For all duplications, the already mentioned fact that domain scores remain constant and close to one as a function of duplication age indicates a strong trend of conserving the domain composition. This has to be compared with the GO similarity analysis on the same sets of duplicates showing a marked trend for divergence in function with increasing duplication age. An explanation of this phenomenon may be the fact that proteins evolve with point mutations affecting one nucleotide at a time. Domain topology can withstand these mutations without changing significantly, but some elementary biochemical properties that define protein function may vary. In other words, point mutation can change protein function without changing their domain composition. It is well known that proteins with identical folds can diverge greatly not only in sequence but also in function [26].

Obviously, this functional divergence cannot exceed the physical possibilities of a domain topology: a kinase domain will never bind to DNA. This is compatible with our observation that GO similarities do not drop to zero, and even very ancient duplicates always retain some degree of functional overlap. Along the same lines, Wapinski and collaborators [24] observe that the functional fates of duplicates rarely diverge with respect to biochemical function, but typically diverge with respect to regulatory control. The typical case when this is known to happen is that of transcription factors [35, where the migration of sequences within the same DNA-binding fold can lead to major changes in the affinity for a given set of sequences, and thus to large variation on the set of regulated targets. More simply, GO term divergence could come to a change of cellular compartment or biological process while performing similar biochemical functions. Also note that the trend of the Molecular function GO taxonomy paralog similarity score with duplication age is weaker than the other two taxonomies, Biological Process and Cel- 
lular Component. We extracted from our set some paralogs that maintain exactly the same domain architecture after duplication, while changing their molecular function, their cellular compartment and/or the biological process in which they are involved (GO term similarity $<0.15$ ). It is the case of BDH1 and SOR1, ancient pre-WGD duplicates (datation I). The first is a butanediol dehydrogenase involved in alcohol metabolic processes, while the second is a sorbitol dehydrogenase involved in hexose metabolism. SOR1 is also a post WGD duplicate (datation E) of XYL2, which encodes for a xylitol dehydrogenase. DIN7 and EXO1 are WGD duplicates, both encoding proteins with nuclease activity involved in DNA repair and replication. However, the first one is mitochondrial and the second is nuclear. Similarly the WGD paralogs SEC14 and YKL091C are both phosphatidylinositol/phosphatidylcholine transfer proteins, but the first performs its function in the cytosol and in the Golgi apparatus while the second is nuclear.

Naturally, the coverage of domains on genomes is only partial, which leaves the question open of whether the observed trends of functional annotations with duplication age are due to modifications in the space of domains that are not visible to our methods. While of course this may happen, it seems unlikely that this can affect the global observed trends, assuming that we are observing an unbiased random sample of the existing structural domains. In other words, if the domains that change their topology during evolution have a fixed probability to be in the set of known domains, this would generate on average a decreasing trend of the domain score with duplication age, which we do not observe. A confirmation of this is given by the fact that removing proteins with gaps (protein sequences of 100 or more aminoacids without an attribution of domain), all the observed trends (figure 4, supplementary figure S4) do not change.

Specificity of the Whole-Genome Duplication. We now revert to the specific features of the whole-genome duplication. Double-sided domain architecture comparison of $S$. cerevisiae WGD paralogs with their $K$. waltii ortholog allows to evaluate asymmetric evolution at the domain level. Comparing with a suitable null model, we found no systematic trend for asymmetry (table 21). This is not unexpected, as domains are much more stable than sequences in evolution, so that, even in presence of accelerated evolution at the sequence level, the fold structure could be conserved.

From the functional viewpoint, we observe that the WGD does not follow a different trend in GO-term similarity between paralogs than expected from its age. Thus, we have to conclude that a "functional burst" correlated to accelerated evolution [20] does not differentiate the global duplication from local ones, or that this trend is not visible from the data available to us.

Partitioning the universe of all $S$. cerevisiae domains in locally and globally duplicated ones yields two sets of WGD and non-WGD domains, that 
can have an intersection, as the same domain can be present in both WGD and non-WGD duplicates. Notably. this intersection is enormously larger than expected from a hypergeometric null model, which can be interpreted as the fact that, within the universe of domains, the main distinction is between domains found or not found in duplications, rather than between domains found in global versus local duplications. Thus, again, whole-genome and local duplications are unified, rather than separated by this trend.

However, the domains of WGD duplicates laying outside common set of duplicable domains remain significant, as they give rise to evident peaks in the frequency of observing a domain in the sets of WGD and non-WGD duplicates. Moreover, they are also significant functionally. Indeed, the disjoint sets of WGD-specific and local-duplication specific domains are enriched for different functional categories. Similar categories are found with a more standard functional analysis on the genes. The domain-based and the Gene Ontology functional analyses agree in underlining functional differences between WGD and non-WGD paralogs. There are several works that proved that WGD paralogs and non-WGD paralogs are similarly biased with respect to codon bias and evolutionary rate, although differing significantly in their functional constituency and in the medium number of interacting partners [24, 28, 29. In agreement with these results, we find that fundamental functions, such as ribosomes and translation are enriched in the WGD while peripheral functions, such as secondary metabolism are enriched for local duplications. The rationale for this result might be that functions related to core biological processes, or in general realized by genes with more entangled genetic interactions are more difficult to replicate by duplicating one part at a time as it happens with local duplications [24]. On the other hand, global moves such as the WGD could release these constraints and allow "recycling" and disentanglement of more elaborate cell machinery.

Finally, we can speculate on the consequences of the fact that the functional dichotomy is also found at the domain level. If it is true that function migrates abundantly, the functional dichotomy of local and global duplicates may emerge from migration of function maintaining similar domain structures. However, this cannot be the only source of differentiation, because in that case the same functional differences would not emerge also from the analysis of WGD and non-WGD specific domains. On the contrary, our result indicate that the dichotomy must be at least in part a result of the "special" protein domains that are only found in local or global duplications.

\section{Methods}

Data Sets. We used the SUPERFAMILY database version 1.69 [7, 15, for the SCOP superfamily domains assignment, and the functional annotation 
of domains. We implemented a $\mathrm{C}$ code to reconstruct the protein domain architectures, as ordered lists of domains and "gaps" (a protein subsequence of 100 AA or more not scored for domains). As a reference for homology assignment we used different homology tools based on sequence alignment and synteny. For sequence-based homology, we referred to Ensembl-Compara (release 50) [16]. For K. waltii-S. cerevisiae WGD duplicates we referred to refs. [8, 36] and to ref. [24]; the latter study was also used for the datation of duplicates.

Homology criteria. Three different homology criteria were used to compare the domain architecture of proteins [17, 32]. Criterion A considers exactly matching architectures. The underlying biological hypothesis is that divergence after duplication does not change the domain architecture of the proteins, implying that divergence between homologs should happen at the sequence/peptide level. Criterion $\mathbf{B}$ relaxes the previous condition, and considers homologous domain architectures that are equal or contain multiple repetition of ordered sets of domains, ignoring possible gap mismatches. Criterion $\mathbf{C}$ further relaxes the above conditions, considering domain architectures as homologous if one contains repeated architecture domain sequences possibly interspaced by gaps or other domains. The code that implements the three criteria is available from the authors upon request.

Domain architecture comparison scores. We defined two different methods to compare proteins in their structural properties. The first "domain score" quantifies the variation in the domains of the two architectures, and is defined as the number of common domains domains between the two architectures, divided by the total number of distinct domains found in both. The domain score measures the number of distinct domain topologies common to the two compared genes, ignoring gaps. It normalizes the score over the total number of different domains contained in the two architectures. The second "architecture score" takes into account the order of appearance of domains in the two architectures and is defined as the length of the longest matching string of domains and gaps between the two architectures, divided by their mean length. The architecture score measures the length (number of ordered domains) of the longest overlapping sequence between the domain architecture of the compared genes, treating gaps as domains (normalized over the mean length of the two architectures). Both scores have a range from 0 (no similarity) to 1 (full similarity). The scores for pairs of WGD, and non-WGD paralogs of different age groups were averaged and histogrammed. To test for asymmetric domain evolution of WGD duplicates, we considered a null model that randomly exchanges the values 
in the hash table containing the two scores between each of the $S$. cerevisiae paralogs and their corresponding $K$. waltii ortholog. The null hypothesis negates the anti-correlation expected in paralog proteins following uneven evolution. The code that implements the two scores is available with the authors.

Domain-based functional analysis. Duplicate proteins with nonempty domain architecture were divided into two disjoint sets of WGD and nonWGD duplicates. The first set, from ref. [36], is composed by 692 S. cerevisiae proteins, estimated to be $62 \%$ of the total WGD paralogs. The second set (1863 proteins) was defined by those proteins coded by a gene with at least one known homolog, from which we removed the other set. Structural domains extracted from the two sets were divided accordingly into three sets: the set $\mathcal{O}$ of domains found in WGD duplicates; the set $\mathcal{P}$ of domains found in non-WGD duplicates; the set $\mathcal{O} \cap \mathcal{P}$ of domains found in at least one member of both protein sets (figure 5). To assess the functional enrichment for WGD and non-WGD paralogs, we implemented a null model based on the hypergeometric distribution, which provides the expected number of domains assigned with function $\mathcal{F}$ belonging either to WGD paralogs or to non-WGD paralogs, using as universe the set of all distinct domains found in $S$. cerevisiae.

Gene Ontology analysis We downloaded the Gene Ontology (GO) annotation DAGs from the GO website (http://www. geneontology .org) and the gene product annotations from the Ensembl database, version 46. We considered a gene annotated to a GO term if it was directly annotated to it or to any of its descendants in the GO tree. We used the SYNERGY algorithm [24] for defining paralogy classes. Orthologs and paralogs were considered different groups. As a reference, 100 pairs of sets were considered, each consisting of 1000 randomly assorted genes with the only constraint that each gene was chosen only once in each pair. For each group we implemented an exact Fisher's test to assess whether a set of genes could be enriched in a certain GO term [37, 38]. Fisher's test gives the probability $P$ of obtaining an equal or greater number of genes annotated to the term in a set made of the same number of genes, but randomly selected. Subsequently, the terms shared by both groups and the exclusive terms (terms present in only one group) were extracted. Finally, we filtered the results retaining only GO terms with $\mathrm{P}$-values $<=10^{-3}$. For each pair of paralogs, we calculated the Lin GO term similarity, by using the GOSim R-package (Version 1.1.5.1) [25]. For each duplication date group we calculated the mean and the standard deviation of the mean of the GO term similarity. 


\section{Acknowledgement}

We would like to thank Hervé Isambert for useful discussions, and Paolo Provero for critical reading of this manuscript.

\section{References}

[1] Rubin GM, Yandell MD, Wortman JR, al (2000) Comparative genomics of the eukaryotes. Science 287:2204-2215.

[2] Koonin EV, Galperin MY (1997) Prokaryotic genomes: the emerging paradigm of genome-based microbiology. Curr Opin Genet Dev 7:75763.

[3] Lander ES, Linton LM, Birren B, al (2001) Initial sequencing and analysis of the human genome. Nature 409:860-921.

[4] McLysaght A, Hokamp K, Wolfe KH (2002) Extensive genomic duplication during early chordate evolution. Nat Genet 31:200-4.

[5] Langkjaer RB, Cliften PF, Johnston M, Piskur J (2003) Yeast genome duplication was followed by asynchronous differentiation of duplicated genes. Nature 421:848-852.

[6] Murzin AG, Brenner SE, Hubbard T, Chothia C (1995) SCOP: a structural classification of proteins database for the investigation of sequences and structures. J Mol Biol 247:536-540.

[7] Gough J, Karplus K, Hughey R, Chothia C (2001) Assignment of Homology to Genome Sequences using a Library of Hidden Markov Models that Represent all Proteins of Known Structure. J Mol Biol 313:903919 .

[8] Kellis M, Birren BW, Lander ES (2004) Proof and evolutionary analysis of ancient genome duplication in the yeast saccharomices cerevisiae. Nature 428:617-624.

[9] Wetlaufer DB (1973) Nucleation, rapid folding, and globular intrachain regions in proteins. Proc Natl Acad Sci U S A 70:697-701.

[10] Richardson JS (1981) The anatomy and taxonomy of protein structure. Adv Protein Chem 34:167-339.

[11] Bork P, Doolittle RF (1992) Proposed acquisition of an animal protein domain by bacteria. Proc Natl Acad Sci U S A 89:8990-4. 
[12] Madera M, Gough J (2002) A comparison of profile hidden Markov model procedures for remote homology detection. Nucleic Acids Research 30:4321-4328.

[13] Orengo CA, Thornton JM (2005) Protein families and their evolution-a structural perspective. Annu Rev Biochem 74:867-900.

[14] Madan Babu M, Teichmann SA (2003) Evolution of transcription factors and the gene regulatory network in Escherichia coli. Nucl Acids Res 31:1234-1244. doi:10.1093/nar/gkg210.

[15] Wilson D, Madera M, Vogel C, Chothia C, Gough J (2007) The superfamily database in 2007: families and functions. Nucleic Acids Res 35:308-313. doi:10.1093/nar/gk1910.

[16] Flicek P, Aken BL, Beal K, Ballester B, Caccamo M, et al. (2008) Ensembl 2008. Nucl Acids Res 36:D707-714. doi:10.1093/nar/gkm988.

[17] Babu MM, Teichmann SA (2004) Gene regulatory network growth by duplication. Nature Genetics 36:492-496.

[18] Cusack BP, Wolfe KH (2007) Not born equal: increased rate asymmetry in relocated and retrotransposed rodent gene duplicates. Mol Biol Evol 24:679-86.

[19] Ohno S (1970) Evolution by gene duplication. London, UK: Allen and Unwin.

[20] Scannell DR, Wolfe KH (2008) A burst of protein sequence evolution and a prolonged period of asymmetric evolution follow gene duplication in yeast. Genome Res 18:137-47.

[21] Byrne KP, Wolfe KH (2007) Consistent patterns of rate asymmetry and gene loss indicate widespread neofunctionalization of yeast genes after whole-genome duplication. Genetics 175:1341-50.

[22] Force A, Lynch M, Pickett FB, Amores A, Yan Yl, et al. (1999) Preservation of Duplicate Genes by Complementary, Degenerative Mutations. Genetics 151:1531-1545.

[23] Lynch M, Force A (2000) The Probability of Duplicate Gene Preservation by Subfunctionalization. Genetics 154:459-473.

[24] Wapinski I, Pfeffer A, Friedman N, Regev A (2007) Natural history and evolutionary principles of gene duplication in fungi. Nature 449:54-61.

[25] Frohlich H, Speer N, Poustka A, BeiSZbarth T (2007) Gosim - an rpackage for computation of information theoretic go similarities between terms and gene products. BMC Bioinformatics 8:166. doi: 10.1186/1471-2105-8-166. 
[26] Carbone MN, Arnold FH (2007) Engineering by homologous recombination: exploring sequence and function within a conserved fold. Current Opinion in Structural Biology 17:454-459.

[27] Rice P, Longden I, Bleasby A (2000) Emboss: The european molecular biology open software suite. Trends in Genetics 16:276 - 277.

[28] Guan Y, Dunham MJ, Troyanskaya OG (2007) Functional Analysis of Gene Duplications in Saccharomyces cerevisiae. Genetics 175:933-943. doi:10.1534/genetics.106.064329.

[29] C DJ, A PD (2005) Do disparate mechanisms of duplication add similar genes to the genome? Trends in Genetics 21:548-551.

[30] Cosentino-Lagomarsino M, Jona P, Bassetti B, Isambert H (2007) Hierarchy and Feedback in the Evolution of E. Coli Transcription Network. Proc Natl Acad Sci U S A 104:5516-20.

[31] Apic G, Gough J, Teichmann SA (2001) Domain combinations in archaeal, eubacterial and eukaryotic proteomes. J Mol Biol 310:311-25.

[32] Moore AD, Bjorklund K, Ekman D, Bornberg-Bauer E, Elofsson A (2008) Arrangements in the modular evolution of proteins. Trends in Biochemical Sciences 33:444-451.

[33] Durrens P, Nikolski M, Sherman D (2008) Fusion and fission of genes define a metric between fungal genomes. PLoS Comput Biol 4:e1000200.

[34] Price MN, Dehal PS, Arkin AP (2008) Horizontal gene transfer and the evolution of transcriptional regulation in Escherichia coli. Genome Biol 9:R4.

[35] Itzkovitz S, Tlusty T, Alon U (2006) Coding limits on the number of transcription factors. BMC Genomics 7:239.

[36] Byrne KP, Wolfe KH (2005) The Yeast Gene Order Browser: combining curated homology and syntenic context reveals gene fate in polyploid species. Genome Res 15:1456-61.

[37] Cora D, Di Cunto F, Provero P, Silengo L, Caselle M (2004) Computational identification of transcription factor binding sites by functional analysis of sets of genes sharing overrep-resented upstream motifs. BMC Bioinformatics 5:57. doi:10.1186/1471-2105-5-57.

[38] Cora D, Herrmann C, Dieterich C, Di Cunto F, Provero P, et al. (2005) $\mathrm{Ab}$ initio identification of putative human transcription factor binding sites by comparative genomics. BMC Bioinformatics 6:110. doi:10. 1186/1471-2105-6-110. 


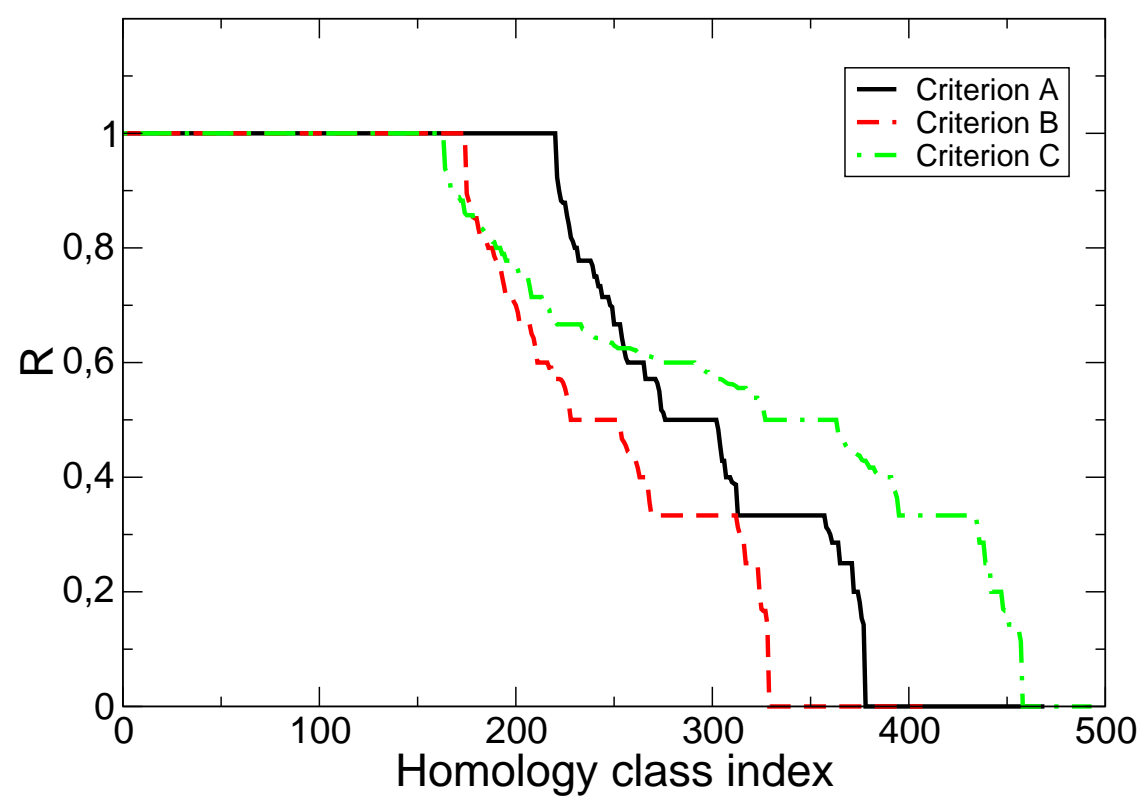

Figure 1: The fraction of architecture homology classes $\mathbf{R}$ not recognized by Ensembl-Compara, plotted for all homology classes, ranked by $\mathrm{R}$ on the $x$-axis. The different lines in the plot refer to homology criteria A (black solid line), B (red dashed line) $\mathbf{C}$ (green dash-dotted line), defined in the text. Criterion $\mathbf{A}$ has the highest number of classes that are entirely not covered by Ensembl-Compara classes. Criterion $\mathbf{C}$, while having the the lowest number of entirely not covered classes, also has the lowest rate of entirely covered ones. 

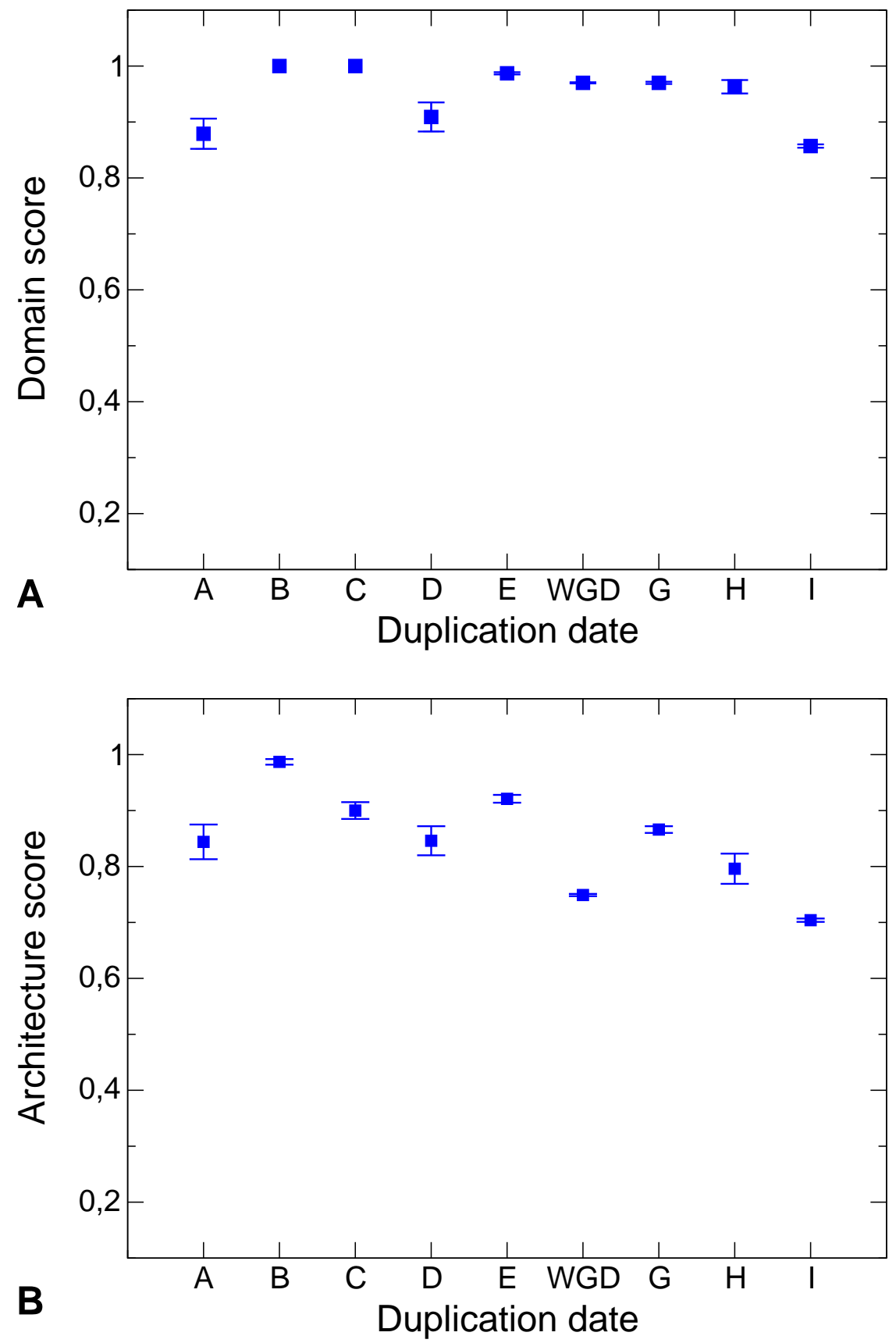

Figure 2: A: Domain score as a function of duplication date. B: Architecture score as a function of duplication date. The duplication refer to SYNERGY duplication age groups from 24]. A, B, C, D and E are post-WGD duplications, while I, H and G are pre-WGD duplications. The value for every date represent the mean over the scores of all duplicate pairs referring to that duplication age group. 

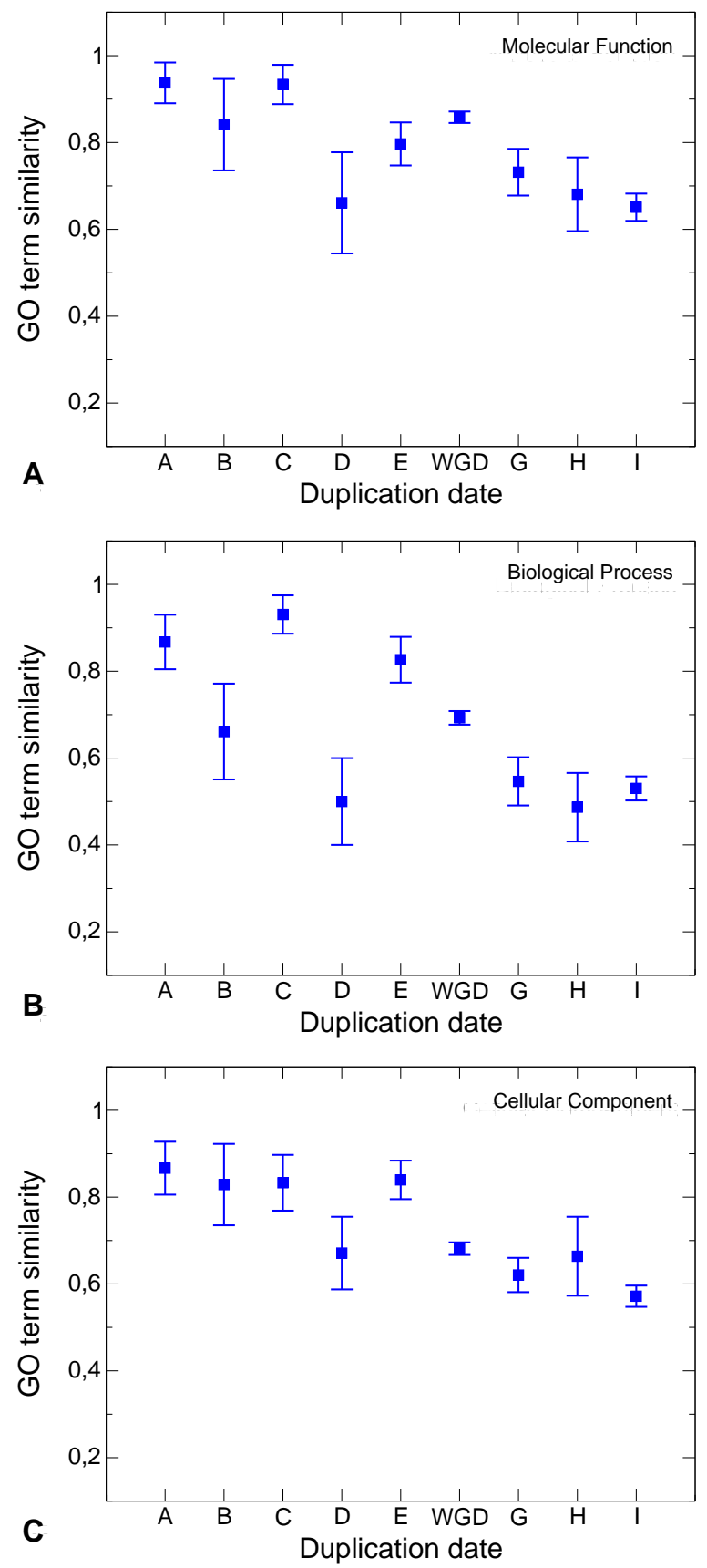

Figure 3: Functional similarity of duplicates and duplication age. The $y$-axes of the plots report mean similarity score (squares) and standard deviation (error bars) between the associated GO terms [25] of duplicates, computed over sets of duplicate pairs belonging to the same age groups ( $x$ axis). The three panels refer to each of the three GO branches: molecular function (A), cellular component (B), biological process (C). Note that in all the plots the GO term similarity values tend to decrease with duplication age. 


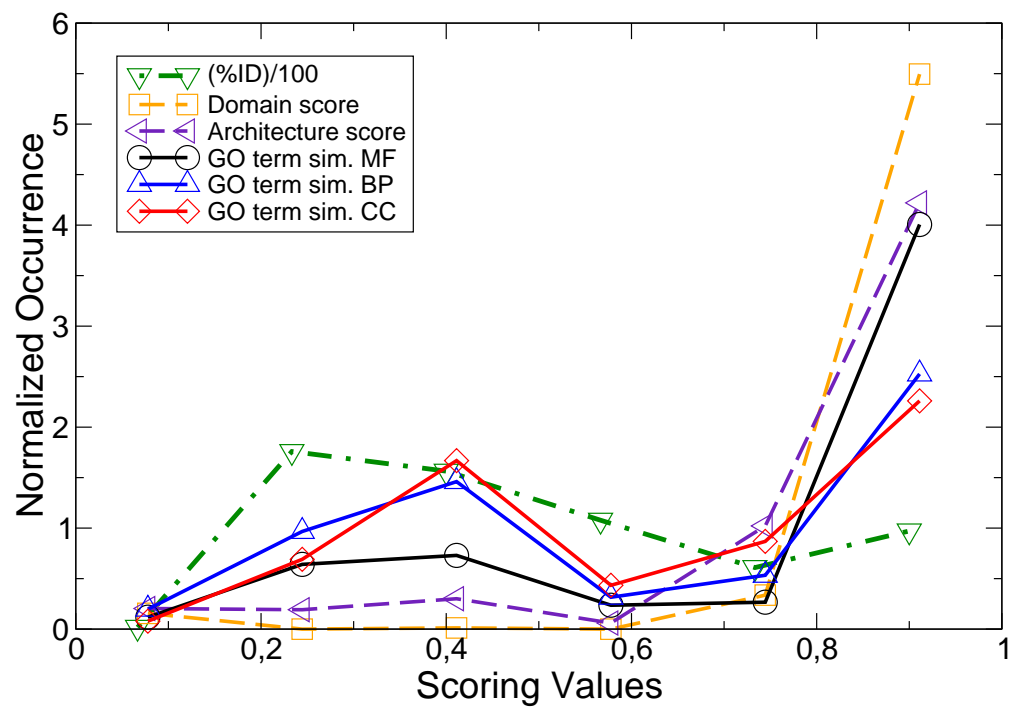

Figure 4: Structural and Functional Divergence of Paralogs. The plot reports histogram over all paralog pairs of domain score (squares), architecture score (left triangles), sequence identity (down triangles) and GO similarity (for all three taxonomies: molecular function, circles, biological process, up triangles, cellular compartment, diamonds). All curves are peaked around the value one, but the highest density values are reached by domain and architecture score curves, while the GO similarity reach lower values at one and develop a secondary peak below 0.5 . This indicates that duplicates tend to maintain domain composition and architecture changing their functions. 


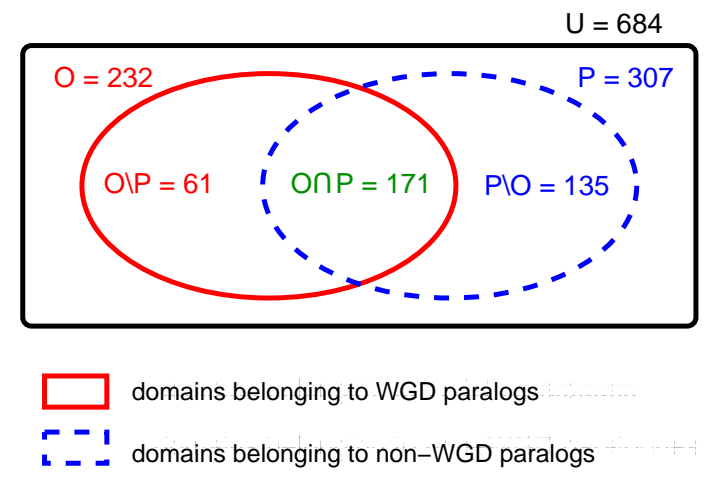

\begin{tabular}{|l|l|}
\hline \multicolumn{1}{|c|}{ Enriched in non-WGD $(\mathbf{z P}, \mathbf{z O})$} & \multicolumn{1}{c|}{ Enriched in WGD $(\mathbf{z P}, \mathbf{z O})$} \\
\hline Secondary metabolites transport $(1.53,-0,83)$ & Coenzyme metabolism $(-1.92,1.09)$ \\
\hline Other regulatory functions $(1.02,-0,94)$ & Ribosomes $(-0.49,1.86)$ \\
\hline Nitrogen metabolism $(2.01,-0,31)$ & Transcription factors $(-1.24,0.34)$ \\
\hline DNA replication and repair $(1.31,-1.78)$ & Cell adhesion $(-1.32,0.50)$ \\
\hline
\end{tabular}

B

Figure 5: A: Venn diagrams of the sets involved in the domainbased functional enrichment analysis. The empirical intersection is 11 standard deviations larger than the mean value provided by an hypergeometric distribution. B: Table summarizing the significantly enriched functional classes for the sets of WGD and non-WGD domains. zP and $\mathrm{zO}$ refer respectively to the Z-score for the non-WGD paralogs analysis and the WGD paralogs analysis (the sets $\mathrm{O} \backslash \mathrm{P}$ and $\mathrm{P} \backslash \mathrm{O}$ in panel A). 


\begin{tabular}{|c|c|c|c|c|}
\hline Homology criterion & Triplets & $(\%)$ over total & Pairs & (\%) over total \\
\hline Kellis et al. & 457 & - & 2609 & - \\
\hline Overlap & 289 & $100 \%$ & 1099 & $100 \%$ \\
\hline Criterion A & 207 & $72 \%$ & 734 & $67 \%$ \\
\hline Criterion B & 239 & $83 \%$ & 836 & $76 \%$ \\
\hline Criterion C & 270 & $92 \%$ & 1010 & $91 \%$ \\
\hline
\end{tabular}

\begin{tabular}{|c|cc|c|}
\hline Homology criterion & Ensembl Compara classes & (\%) over total \\
\hline Ensembl & 672 & - \\
\hline Overlap & 470 & $100 \%$ \\
\hline Criterion A & 301 & $64 \%$ \\
\hline Criterion B & 347 & $74 \%$ \\
\hline Criterion C & 403 & $86 \%$ \\
\hline
\end{tabular}

Table 1: Comparison of classes obtained with domain-based homology criteria and homology classes built with WGD duplicates and their orthologs [8] (upper panel) and paralogs relations provided by Ensembl-Compara (lower panel,[16]). For both tables, the first row of the shows the number of genes in sequenced-based homology classes. The second row reads the result of the intersection of these data with the architecture databases. The following three rows report the total and the relative fraction of the number of triplets and pairs found in the homology classes with criteria $\mathbf{A}, \mathbf{B}$, and $\mathbf{C}$. 


\begin{tabular}{ccccc}
\hline \multicolumn{5}{c}{ Domain Score Comparison } \\
\hline & Empirical & Randomized & Difference (\%) & P-value \\
\hline F2 triplets & $80 \%$ & $73 \%$ & $-7 \%$ & $<10^{-4}$ \\
F1 triplets & $11 \%$ & $15 \%$ & $-4 \%$ & $<10^{-4}$ \\
\hline \multicolumn{5}{c}{ Architecture Score Comparison } \\
\hline & Empirical & Randomized & Difference (\%) & P-value \\
\hline F2 triplets & $65 \%$ & $53 \%$ & $-12 \%$ & $<10^{-4}$ \\
F1 triplets & $16 \%$ & $50 \%$ & $+34 \%$ & $<10^{-4}$ \\
\hline
\end{tabular}

Table 2: Quantification of uneven architecture divergence between duplicates. The table shows experimental and null-model relative frequencies of WGD paralogs in S. cerevisiae having identical architecture to their WGD ortholog in K. waltii according to the domain (upper panel) and architecture (lower panel) scores. The first two rows of each panel show the statistics restricted to the F2 and F1 triplets. 


\section{Supporting Information}
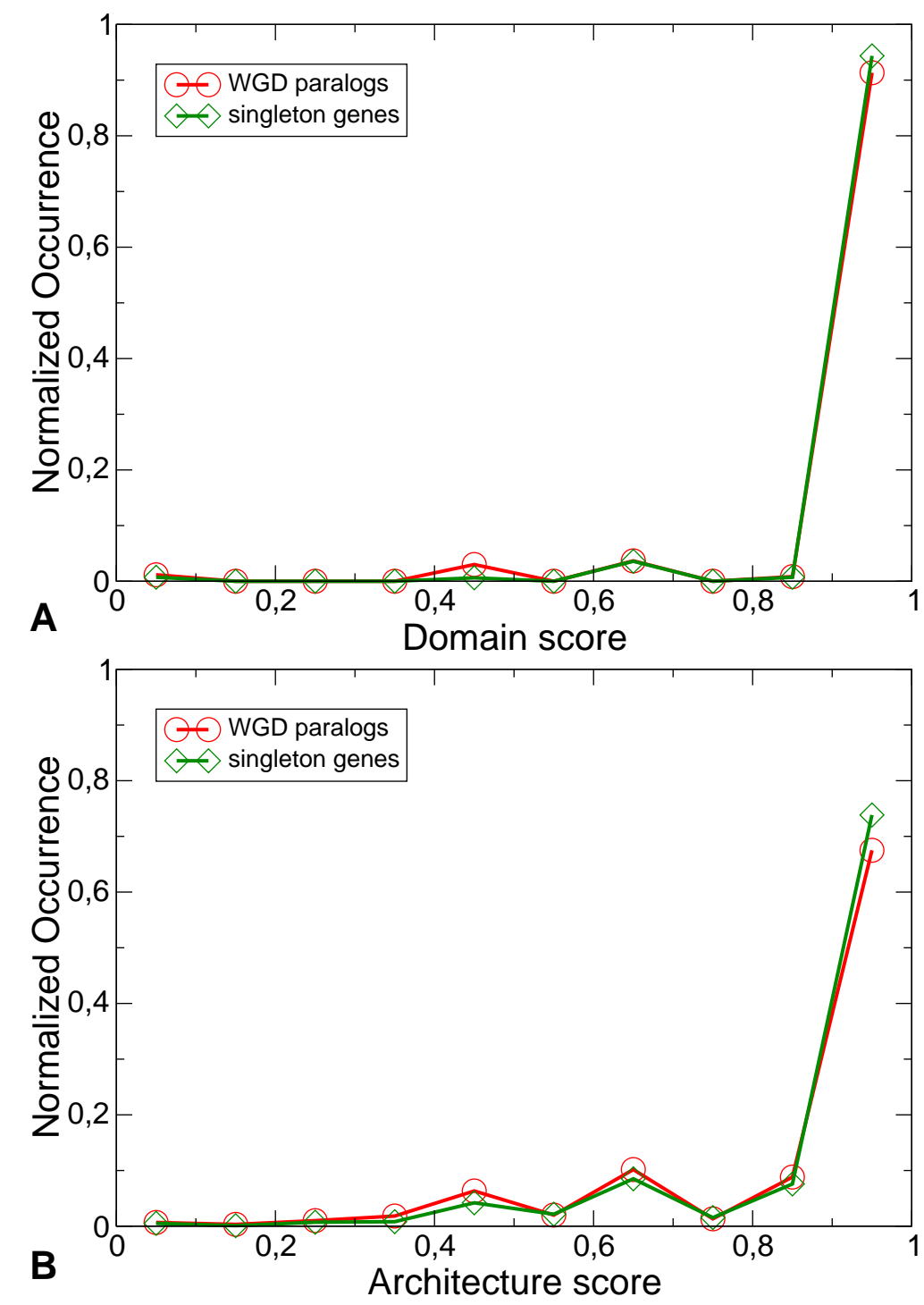

Figure S1: WGD duplicates and single-copy orthologs show similar domain architecture divergence. The plots report the histograms of domain score (A) and architecture score (B), evaluated in pairs of orthologs of S. cerevisiae and K. waltii for WGD duplicates and single copy S. cerevisiae-K. waltii orthologs. 

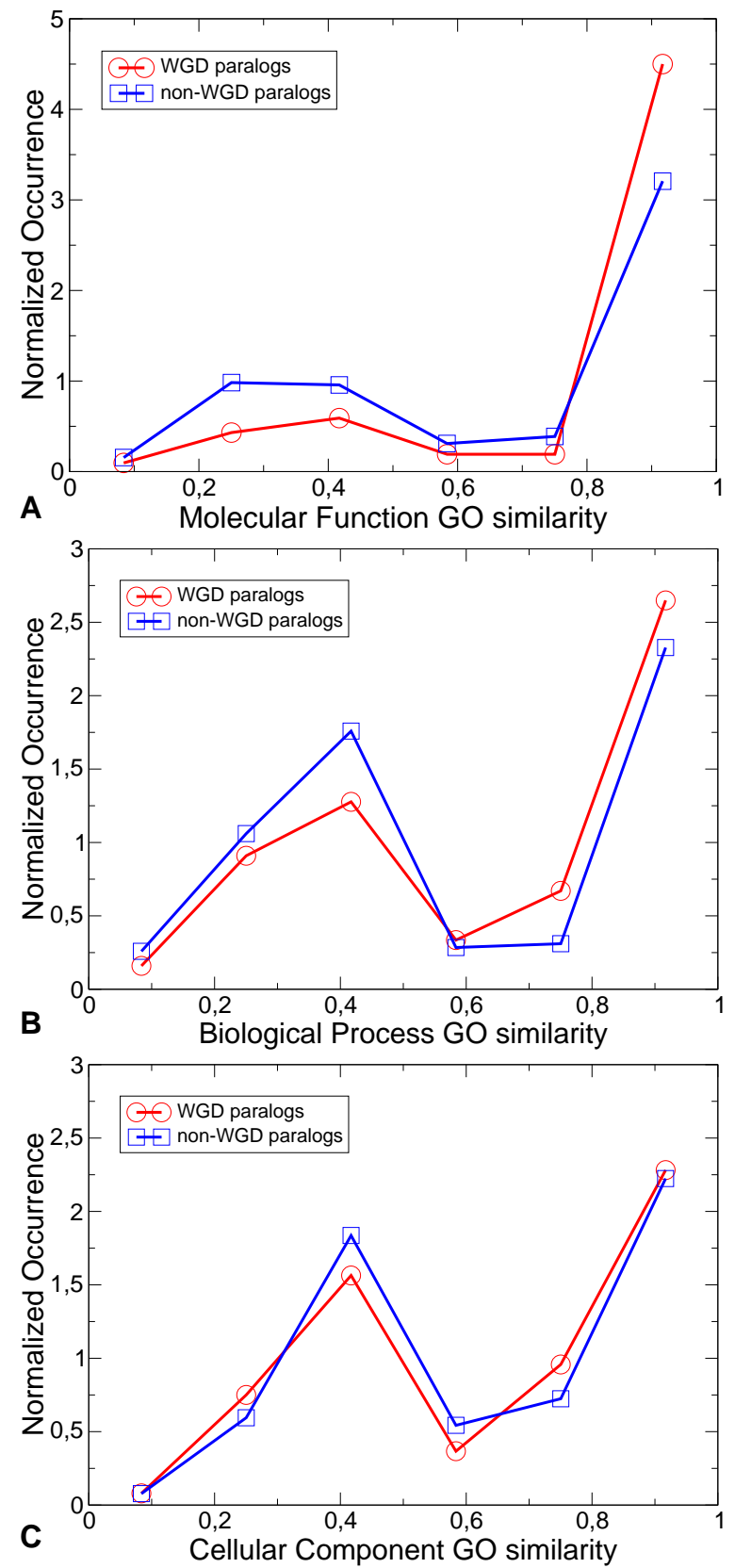

Figure S2: Functional Similarity of WGD paralogs and non-WGD paralogs. Normalized histograms of the Gene Ontology similarity between WGD and non-WGD duplicate pairs for the GO branches molecular function (A), biological process (B), cellular component (C). For all the three branches, WGD paralogs tend to have higher GO similarity scores than non-WGD paralogs. 

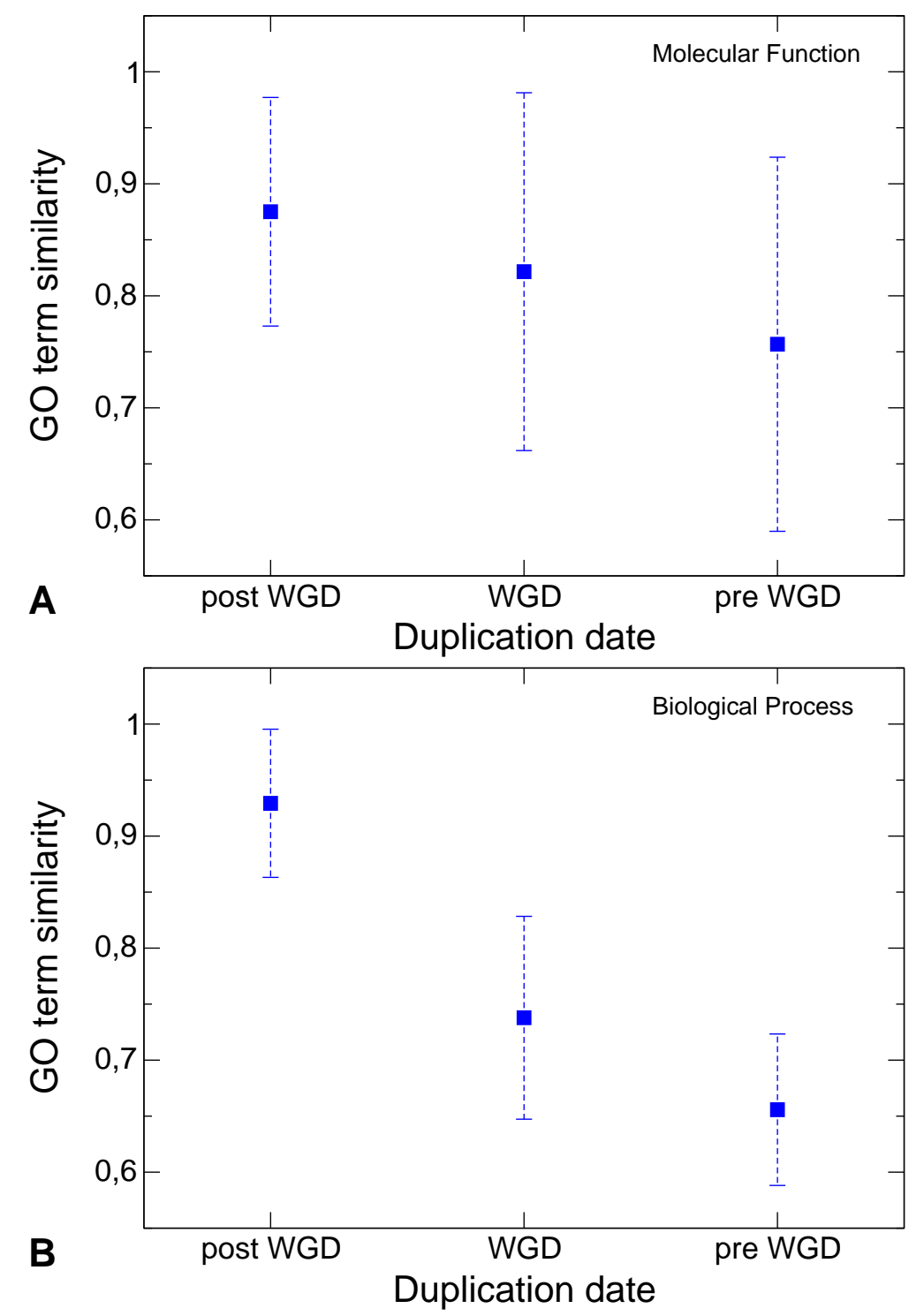

Figure S3: Functional similarity of duplicates versus duplication age for manually curated GO annotations. The plots report the mean (squares) and the standard deviation (error bars) of the GOsim similarity score between duplicates of the same age groups. The analysis was restricted only to the genes with experimental manually curated GO terms, grouping pre- and post-WGD duplication to gather sufficient statistics. This comparison is made for the GO branches: Biological Process (A), Molecular Function (B). 


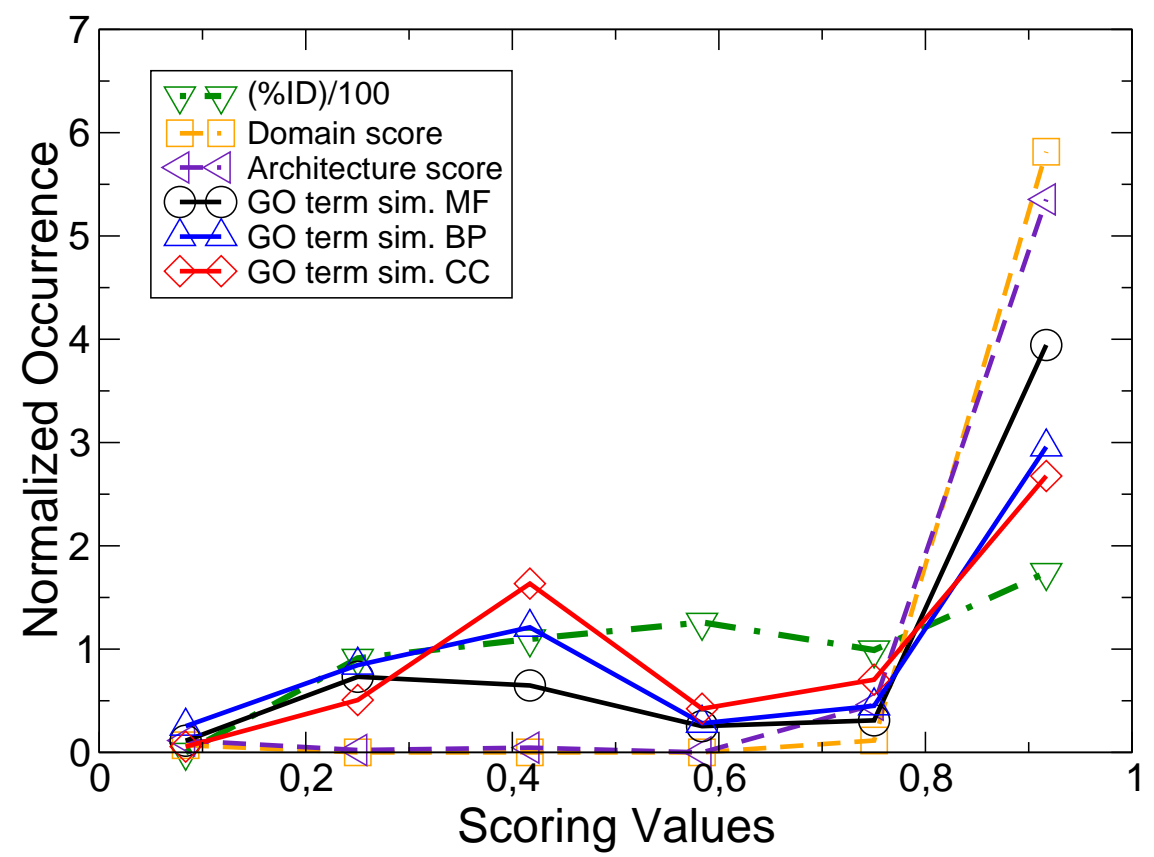

Figure S4: Structural and functional divergence of paralogs with no gaps in the domain architecture. The plot reports histograms of sequence ID\% retrieved from alignment, domain score, architecture score and GO term similarity (for all three branches) for all the paralog pairs with both proteins with by domain. Despite of this restriction we retrieve the same results shown in Figure 4 of the main text. 


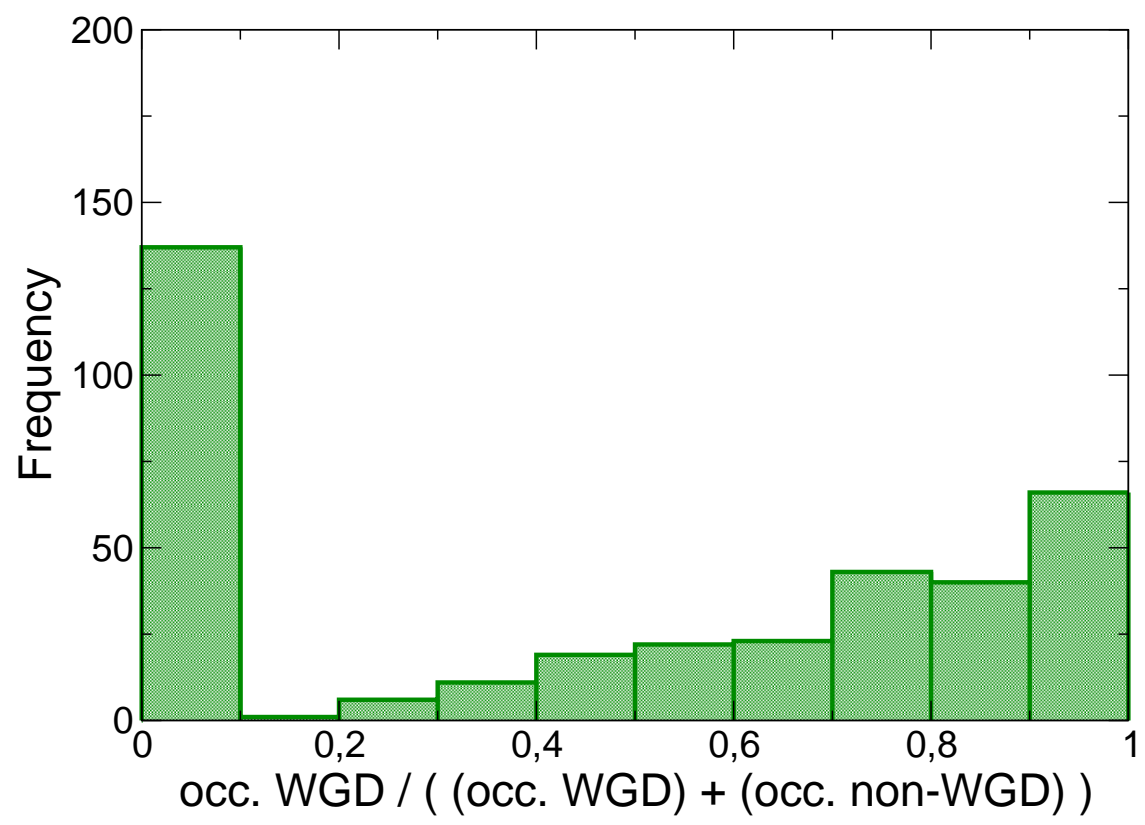

Figure S5: Occurrence of domain topologies in WGD vs non-WGD duplicates. For each SCOP domain, we calculated its occurrence in WGD proteins and non-WGD duplicates (normalized by the sizes of these two duplicate sets). The plot reports the histogram of the relative weight of occurrence of WGD duplicates, indicating the separation of two populations of domain topologies: domain topologies that appear in local duplications only (peak at zero), and those that appear in both the WGD and local duplications, having a preference towards the WGD (peak at one). 


\begin{tabular}{|c|c|c|c|}
\hline \multicolumn{4}{|c|}{ Gene Ontology terms exclusive of WGD-Paralogs } \\
\hline GO term & Number of genes & P-value & annotation \\
\hline GO:0005737 & 571 & $3.62 \mathrm{e}-22$ & cytoplasm \\
\hline GO:0009987 & 647 & $1.72 \mathrm{e}-21$ & cellular process \\
\hline GO:0005622 & 675 & $8.80 \mathrm{e}-19$ & intracellular \\
\hline GO:0044424 & 668 & $1.10 \mathrm{e}-17$ & intracellular part \\
\hline GO:0005830 & 56 & $1.60 \mathrm{e}-17$ & cytosolic ribosome (sensu Eukaryota) \\
\hline GO:0005840 & 97 & $6.97 \mathrm{e}-16$ & ribosome \\
\hline GO:0005575 & 740 & $5.40 \mathrm{e}-15$ & cellular component \\
\hline GO:0005829 & 92 & $5.57 \mathrm{e}-15$ & cytosol \\
\hline GO:0044445 & 58 & $1.12 \mathrm{e}-14$ & cytosolic part \\
\hline GO:0044464 & 737 & $2.86 \mathrm{e}-14$ & cell part \\
\hline GO:0005623 & 737 & $3.11 \mathrm{e}-14$ & cell \\
\hline GO:0016773 & 62 & $4.53 \mathrm{e}-14$ & phosphotransferase activity, alcohol group as acceptor \\
\hline GO:0004674 & 49 & $5.75 \mathrm{e}-14$ & protein serine/threonine kinase activity \\
\hline GO:0009059 & 138 & $6.01 \mathrm{e}-14$ & macromolecule biosynthetic process \\
\hline GO:0004672 & 49 & $2.19 \mathrm{e}-13$ & protein kinase activity \\
\hline GO:0016301 & 66 & $4.33 \mathrm{e}-13$ & kinase activity \\
\hline GO:0003735 & 68 & $7.94 \mathrm{e}-13$ & structural constituent of ribosome \\
\hline GO:0009058 & 203 & $1.13 \mathrm{e}-12$ & biosynthetic process \\
\hline GO:0044262 & 69 & $1.45 \mathrm{e}-12$ & cellular carbohydrate metabolic process \\
\hline GO:0004713 & 42 & $3.62 \mathrm{e}-12$ & protein-tyrosine kinase activity \\
\hline GO:0065007 & 228 & $4.49 \mathrm{e}-12$ & biological regulation \\
\hline GO:0005488 & 536 & $6.77 \mathrm{e}-12$ & binding \\
\hline GO:0043284 & 31 & $7.92 \mathrm{e}-12$ & biopolymer biosynthetic process \\
\hline GO:0000271 & 25 & $7.93 \mathrm{e}-12$ & polysaccharide biosynthetic process \\
\hline GO:0006468 & 47 & $9.56 \mathrm{e}-12$ & protein amino acid phosphorylation \\
\hline GO:0044444 & 383 & $3.28 \mathrm{e}-11$ & cytoplasmic part \\
\hline GO:0007154 & 85 & $5.55 \mathrm{e}-11$ & cell communication \\
\hline GO:0007165 & 80 & $9.09 \mathrm{e}-11$ & signal transduction \\
\hline GO:0005843 & 26 & $1.60 \mathrm{e}-10$ & cytosolic small ribosomal subunit (sensu Eukaryota) \\
\hline GO:0006412 & 94 & $3.37 \mathrm{e}-10$ & translation \\
\hline GO:0032502 & 106 & $3.71 \mathrm{e}-10$ & developmental process \\
\hline GO:0016051 & 33 & $5.93 \mathrm{e}-10$ & carbohydrate biosynthetic process \\
\hline GO:0033279 & 56 & $1.01 \mathrm{e}-09$ & ribosomal subunit \\
\hline GO:0008152 & 520 & $1.6 \mathrm{e}-09$ & metabolic process \\
\hline GO:0050789 & 187 & $1.74 \mathrm{e}-09$ & regulation of biological process \\
\hline GO:0046164 & 27 & $2.35 \mathrm{e}-09$ & alcohol catabolic process \\
\hline GO:0006112 & 20 & $2.38 \mathrm{e}-09$ & energy reserve metabolic process \\
\hline GO:0044249 & 152 & $3.72 \mathrm{e}-09$ & cellular biosynthetic process \\
\hline GO:0044260 & 244 & $3.99 \mathrm{e}-09$ & cellular macromolecule metabolic process \\
\hline GO:0016052 & 30 & $5.11 \mathrm{e}-09$ & carbohydrate catabolic process \\
\hline GO:0044275 & 30 & $5.11 \mathrm{e}-09$ & cellular carbohydrate catabolic process \\
\hline GO:0050794 & 181 & $6.51 \mathrm{e}-09$ & regulation of cellular process \\
\hline GO:0016310 & 56 & $9.85 \mathrm{e}-09$ & phosphorylation \\
\hline GO:0005842 & 27 & $1.09 \mathrm{e}-08$ & cytosolic large ribosomal subunit (sensu Eukaryota) \\
\hline GO:0044237 & 485 & $1.35 \mathrm{e}-08$ & cellular metabolic process \\
\hline GO:0006739 & 13 & $1.38 \mathrm{e}-08$ & NADP metabolic process \\
\hline GO:0019320 & 24 & $1.41 \mathrm{e}-08$ & hexose catabolic process \\
\hline GO:0044264 & 27 & $1.55 \mathrm{e}-08$ & cellular polysaccharide metabolic process \\
\hline GO:0005976 & 27 & $1.55 \mathrm{e}-08$ & polysaccharide metabolic process \\
\hline GO:0044238 & 478 & $1.57 \mathrm{e}-08$ & primary metabolic process \\
\hline GO:0005516 & 11 & $1.86 \mathrm{e}-08$ & calmodulin binding \\
\hline GO:0032989 & 62 & $1.91 \mathrm{e}-08$ & cellular structure morphogenesis \\
\hline GO:0000902 & 62 & $1.91 \mathrm{e}-08$ & cell morphogenesis \\
\hline GO:0006007 & 23 & $2.22 \mathrm{e}-08$ & glucose catabolic process \\
\hline GO:0009250 & 16 & $2.50 \mathrm{e}-08$ & glucan biosynthetic process \\
\hline GO:0006006 & 30 & $2.56 \mathrm{e}-08$ & glucose metabolic process \\
\hline GO:0009653 & 62 & $2.63 \mathrm{e}-08$ & anatomical structure morphogenesis \\
\hline GO:0005198 & 81 & $2.95 \mathrm{e}-08$ & structural molecule activity \\
\hline GO:0005978 & 12 & $2.98 \mathrm{e}-08$ & glycogen biosynthetic process \\
\hline GO:0006796 & 65 & $3.81 \mathrm{e}-08$ & phosphate metabolic process \\
\hline GO:0006793 & 65 & $3.81 \mathrm{e}-08$ & phosphorus metabolic process \\
\hline GO:0006066 & 52 & $6.20 \mathrm{e}-08$ & alcohol metabolic process \\
\hline GO:0048856 & 62 & $7.67 \mathrm{e}-08$ & anatomical structure development \\
\hline GO:0007242 & 53 & $7.81 \mathrm{e}-08$ & intracellular signaling cascade \\
\hline GO:0046365 & 24 & $9.47 \mathrm{e}-08$ & monosaccharide catabolic process \\
\hline GO:0019318 & 34 & $9.49 \mathrm{e}-08$ & hexose metabolic process \\
\hline GO:0030529 & 107 & $1.25 \mathrm{e}-07$ & ribonucleoprotein complex \\
\hline GO:0006073 & 20 & $1.31 \mathrm{e}-07$ & glucan metabolic process \\
\hline GO:0007265 & 23 & $1.54 \mathrm{e}-07$ & Ras protein signal transduction \\
\hline GO:0005977 & 16 & $1.56 \mathrm{e}-07$ & glycogen metabolic process \\
\hline GO:0065008 & 74 & $1.59 \mathrm{e}-07$ & regulation of biological quality \\
\hline GO:0006740 & 11 & $1.78 \mathrm{e}-07$ & NADPH regeneration \\
\hline GO:0006897 & 28 & $2.25 \mathrm{e}-07$ & endocytosis \\
\hline GO:0010324 & 30 & $2.48 \mathrm{e}-07$ & membrane invagination \\
\hline GO:0019843 & 17 & $3.02 \mathrm{e}-07$ & rRNA binding \\
\hline GO:0050793 & 11 & $4.48 \mathrm{e}-07$ & regulation of developmental process \\
\hline GO:0016772 & 77 & $5.36 \mathrm{e}-07$ & transferase activity, transferring phosphorus-containing groups \\
\hline GO:0005933 & 40 & $6.06 \mathrm{e}-07$ & cellular bud \\
\hline GO:0005996 & 34 & $6.13 \mathrm{e}-07$ & monosaccharide metabolic process \\
\hline GO:0030955 & 9 & $7.98 \mathrm{e}-07$ & potassium ion binding \\
\hline GO:0051726 & 44 & $9.88 \mathrm{e}-07$ & regulation of cell cycle \\
\hline
\end{tabular}




\begin{tabular}{|c|c|c|c|}
\hline GO:0000074 & 44 & $9.88 \mathrm{e}-07$ & regulation of progression through cell cycle \\
\hline GO:0006098 & 10 & $1.03 \mathrm{e}-06$ & pentose-phosphate shunt \\
\hline GO:0009117 & 41 & $1.15 \mathrm{e}-06$ & nucleotide metabolic process \\
\hline GO:0007264 & 34 & $1.76 \mathrm{e}-06$ & small GTPase mediated signal transduction \\
\hline GO:0005979 & 6 & $2.99 \mathrm{e}-06$ & regulation of glycogen biosynthetic process \\
\hline GO:0051278 & 12 & $3.25 \mathrm{e}-06$ & chitin- and beta-glucan-containing cell wall polysaccharide biosynthetic process \\
\hline GO:0008360 & 8 & $5.04 \mathrm{e}-06$ & regulation of cell shape \\
\hline GO:0006038 & 8 & $5.04 \mathrm{e}-06$ & cell wall chitin biosynthetic process \\
\hline GO:0022603 & 8 & $5.04 \mathrm{e}-06$ & regulation of anatomical structure morphogenesis \\
\hline GO:0022604 & 8 & $5.04 \mathrm{e}-06$ & regulation of cell morphogenesis \\
\hline GO:0006769 & 17 & $5.74 \mathrm{e}-06$ & nicotinamide metabolic process \\
\hline GO:0044267 & 220 & $7.05 \mathrm{e}-06$ & cellular protein metabolic process \\
\hline GO:0015935 & 26 & $7.80 \mathrm{e}-06$ & small ribosomal subunit \\
\hline GO:0005935 & 31 & $8.82 \mathrm{e}-06$ & cellular bud neck \\
\hline GO:0019362 & 17 & $1.16 \mathrm{e}-05$ & pyridine nucleotide metabolic process \\
\hline GO:0006031 & 9 & $1.29 \mathrm{e}-05$ & chitin biosynthetic process \\
\hline GO:0006037 & 8 & $1.35 \mathrm{e}-05$ & cell wall chitin metabolic process \\
\hline GO:0000028 & 8 & $1.35 \mathrm{e}-05$ & ribosomal small subunit assembly and maintenance \\
\hline GO:0048610 & 36 & $1.53 \mathrm{e}-05$ & reproductive cellular process \\
\hline GO:0022413 & 36 & $1.53 \mathrm{e}-05$ & reproductive process in single-celled organism \\
\hline GO:0030427 & 37 & $1.59 \mathrm{e}-05$ & site of polarized growth \\
\hline GO:0016192 & 70 & $1.61 \mathrm{e}-05$ & vesicle-mediated transport \\
\hline GO:0005934 & 18 & $1.83 \mathrm{e}-05$ & cellular bud tip \\
\hline GO:0005498 & 6 & $1.88 \mathrm{e}-05$ & sterol carrier activity \\
\hline GO:0005496 & 6 & $1.88 \mathrm{e}-05$ & steroid binding \\
\hline GO:0032934 & 6 & $1.88 \mathrm{e}-05$ & sterol binding \\
\hline GO:0006887 & 17 & $2.22 \mathrm{e}-05$ & exocytosis \\
\hline GO:0015934 & 30 & $2.95 \mathrm{e}-05$ & large ribosomal subunit \\
\hline GO:0008361 & 33 & $3.01 \mathrm{e}-05$ & regulation of cell size \\
\hline GO:0015980 & 36 & $3.91 \mathrm{e}-05$ & energy derivation by oxidation of organic compounds \\
\hline GO:0009272 & 13 & $3.91 \mathrm{e}-05$ & chitin- and beta-glucan-containing cell wall biogenesis \\
\hline GO:0040007 & 34 & $4.31 \mathrm{e}-05$ & growth \\
\hline GO:0065009 & 21 & $4.50 \mathrm{e}-05$ & regulation of a molecular function \\
\hline GO:0042546 & 13 & $5.74 \mathrm{e}-05$ & cell wall biogenesis \\
\hline GO:0006665 & 12 & $6.26 \mathrm{e}-05$ & sphingolipid metabolic process \\
\hline GO:0010383 & 8 & $6.56 \mathrm{e}-05$ & cell wall polysaccharide metabolic process \\
\hline GO:0030011 & 6 & $6.75 \mathrm{e}-05$ & maintenance of cell polarity \\
\hline GO:0006869 & 14 & $7.15 \mathrm{e}-05$ & lipid transport \\
\hline GO:0050790 & 20 & $7.36 \mathrm{e}-05$ & regulation of catalytic activity \\
\hline GO:0031505 & 15 & $8.24 \mathrm{e}-05$ & chitin- and beta-glucan-containing cell wall organization and biogenesis \\
\hline GO:0006042 & 9 & $8.97 \mathrm{e}-05$ & glucosamine biosynthetic process \\
\hline GO:0006045 & 9 & $8.97 \mathrm{e}-05$ & $\mathrm{~N}$-acetylglucosamine biosynthetic process \\
\hline GO:0046349 & 9 & $8.97 \mathrm{e}-05$ & amino sugar biosynthetic process \\
\hline GO:0006893 & 12 & $9.31 \mathrm{e}-05$ & Golgi to plasma membrane transport \\
\hline
\end{tabular}

Table S1: Gene Ontology terms exclusive of WGD paralogs. The table reports the results of the enrichment analysis for Gene Ontology terms exclusive of non-WGD duplicates, with populations of functional categories (column two) and P-values from hypergeometric testing (column three).

\begin{tabular}{llll}
\hline & \multicolumn{3}{c}{ Gene Ontology terms } \\
\hline GO term & Number of genes & P-value & annotation \\
\hline GO:0022891 & 60 & $4.99 \mathrm{e}-16$ & substrate-specific transmembrane transporter activity \\
GO:0022857 & 64 & $6.24 \mathrm{e}-16$ & transmembrane transporter activity \\
GO:0022892 & 65 & $1.36 \mathrm{e}-13$ & substrate-specific transporter activity \\
GO:0005215 & 71 & $2.38 \mathrm{e}-13$ & transporter activity \\
GO:0005353 & 11 & $4.78 \mathrm{e}-11$ & fructose transmembrane transporter activity \\
GO:0015578 & 11 & $4.78 \mathrm{e}-11$ & mannose transmembrane transporter activity \\
GO:0005355 & 11 & $1.44 \mathrm{e}-10$ & glucose transmembrane transporter activity \\
GO:0015149 & 11 & $3.86 \mathrm{e}-10$ & hexose transmembrane transporter activity \\
GO:0015145 & 11 & $3.86 \mathrm{e}-10$ & monosaccharide transmembrane transporter activity \\
GO:0015291 & 25 & $1.17 \mathrm{e}-09$ & secondary active transmembrane transporter activity \\
GO:0015293 & 19 & $1.36 \mathrm{e}-09$ & symporter activity \\
GO:0022804 & 35 & $3.71 \mathrm{e}-09$ & active transmembrane transporter activity \\
GO:0015171 & 14 & $1.02 \mathrm{e}-08$ & amino acid transmembrane transporter activity \\
GO:0015837 & 17 & $1.13 \mathrm{e}-08$ & amine transport \\
GO:0051119 & 14 & $1.55 \mathrm{e}-08$ & sugar transmembrane transporter activity \\
GO:0005351 & 14 & $1.55 \mathrm{e}-08$ & sugar:hydrogen ion symporter activity \\
GO:0005342 & 19 & $1.83 \mathrm{e}-08$ & organic acid transmembrane transporter activity \\
GO:0046943 & 18 & $3.04 \mathrm{e}-08$ & carboxylic acid transmembrane transporter activity \\
GO:0015144 & 14 & $3.42 \mathrm{e}-08$ & carbohydrate transmembrane transporter activity \\
GO:0006865 & 15 & $4.90 \mathrm{e}-08$ & amino acid transport \\
GO:0046942 & 19 & $5 \mathrm{e}-08$ & carboxylic acid transport \\
GO:0015849 & 19 & $6.35 \mathrm{e}-08$ & organic acid transport \\
GO:0000023 & 8 & $7.87 \mathrm{e}-08$ & maltose metabolic process \\
GO:0008615 & 8 & $7.87 \mathrm{e}-08$ & pyridoxine biosynthetic process
\end{tabular}




\begin{tabular}{llll} 
GO:0042819 & 8 & $7.87 \mathrm{e}-08$ & vitamin B6 biosynthetic process \\
GO:0008614 & 8 & $1.93 \mathrm{e}-07$ & pyridoxine metabolic process \\
GO:0042816 & 8 & $1.94 \mathrm{e}-07$ & vitamin B6 metabolic process \\
GO:0009277 & 19 & $1.42 \mathrm{e}-06$ & chitin- and beta-glucan-containing cell wall \\
GO:0048503 & 13 & $3.21 \mathrm{e}-06$ & GPI anchor binding \\
GO:0015205 & 6 & $9.08 \mathrm{e}-06$ & nucleobase transmembrane transporter activity \\
GO:0015174 & 6 & $9.08 \mathrm{e}-06$ & basic amino acid transmembrane transporter activity \\
GO:0042402 & 6 & $9.084 \mathrm{e}-06$ & biogenic amine catabolic process \\
GO:0016020 & 168 & $1.22 \mathrm{e}-05$ & membrane \\
GO:0005984 & 8 & $1.29 \mathrm{e}-05$ & disacharide metabolic process \\
GO:0015075 & 29 & $1.82 \mathrm{e}-05$ & ion transmembrane transporter activity \\
GO:0042219 & 6 & $3.59 \mathrm{e}-05$ & amino acid derivative catabolic process \\
GO:0015175 & 5 & $4.20 \mathrm{e}-05$ & neutral amino acid transmembrane transporter activity \\
GO:0030976 & 5 & $4.20 \mathrm{e}-05$ & thiamin pyrophosphate binding \\
GO:0019660 & 5 & $4.20 \mathrm{e}-05$ & glycolytic fermentation \\
GO:0006559 & 4 & $6.82 \mathrm{e}-05$ & L-phenylalanine catabolic process \\
GO:0031224 & 124 & $7.03 \mathrm{e}-05$ & intrinsic to membrane \\
GO:0030287 & 5 & $8.98 \mathrm{e}-05$ & cell wall-bounded periplasmic space \\
GO:0009083 & 5 & $8.98 \mathrm{e}-05$ & branched chain family amino acid catabolic process \\
GO:0044270 & 9 & $9.37 \mathrm{e}-05$ & nitrogen compound catabolic process \\
GO:0009310 & 9 & $9.37 \mathrm{e}-05$ & amine catabolic process \\
GO:0016021 & 123 & $9.81 \mathrm{e}-05$ & integral to membrane \\
\hline
\end{tabular}

Table S2: Gene Ontology terms exclusively found in non-WGD

Paralogs. The table reports the results of the enrichment analysis for Gene Ontology terms exclusive of non-WGD duplicates, with populations of functional categories (column two) and P-values from hypergeometric testing (column three). 


\begin{tabular}{|c|c|c|}
\hline \multicolumn{3}{|c|}{ SCOP superfamily domain occurrence } \\
\hline Domain & Occurrence in WGD proteins & Occurrence in non-WGD proteins \\
\hline 46561 & 2 & 0 \\
\hline 46565 & 0 & 16 \\
\hline 46579 & 0 & 7 \\
\hline 46589 & 0 & 2 \\
\hline 46626 & 2 & 0 \\
\hline 46689 & 8 & 14 \\
\hline 46774 & 0 & 2 \\
\hline 46785 & 8 & 13 \\
\hline 46906 & 2 & 0 \\
\hline 46934 & 2 & 3 \\
\hline 46938 & 2 & 2 \\
\hline 46946 & 2 & 1 \\
\hline 46955 & 0 & 2 \\
\hline 46977 & 2 & 0 \\
\hline 47060 & 0 & 2 \\
\hline 47072 & 0 & 2 \\
\hline 47095 & 4 & 3 \\
\hline 47113 & 2 & 22 \\
\hline 47212 & 2 & 0 \\
\hline 47240 & 2 & 1 \\
\hline 47323 & 2 & 2 \\
\hline 47370 & 4 & 2 \\
\hline 47459 & 0 & 8 \\
\hline 47473 & 2 & 10 \\
\hline 47576 & 0 & 2 \\
\hline 47592 & 4 & 0 \\
\hline 47616 & 0 & 5 \\
\hline 47661 & 2 & 3 \\
\hline 47672 & 1 & 0 \\
\hline 47694 & 0 & 2 \\
\hline 47769 & 2 & 2 \\
\hline 47807 & 2 & 1 \\
\hline 47819 & 0 & 2 \\
\hline 47923 & 4 & 5 \\
\hline 47954 & 10 & 10 \\
\hline 47973 & 0 & 2 \\
\hline 48019 & 0 & 4 \\
\hline 48065 & 2 & 2 \\
\hline 48097 & 0 & 2 \\
\hline 48140 & 2 & 0 \\
\hline 48150 & 2 & 1 \\
\hline 48168 & 2 & 0 \\
\hline 48179 & 2 & 5 \\
\hline 48208 & 2 & 6 \\
\hline 48225 & 0 & 2 \\
\hline 48239 & 0 & 4 \\
\hline 48256 & 2 & 1 \\
\hline 48264 & 0 & 3 \\
\hline 48317 & 2 & 4 \\
\hline 48334 & 0 & 2 \\
\hline 48350 & 6 & 4 \\
\hline 48366 & 2 & 1 \\
\hline 48371 & 8 & 57 \\
\hline 48403 & 6 & 6 \\
\hline 48425 & 2 & 2 \\
\hline 48431 & 1 & 0 \\
\hline 48439 & 0 & 6 \\
\hline 48445 & 2 & 0 \\
\hline 48452 & 6 & 24 \\
\hline 48464 & 6 & 6 \\
\hline 48557 & 0 & 3 \\
\hline 48576 & 0 & 3 \\
\hline 48592 & 0 & 6 \\
\hline 48613 & 0 & 5 \\
\hline 48695 & 2 & 0 \\
\hline 49348 & 2 & 0 \\
\hline 49354 & 2 & 0 \\
\hline 49447 & 0 & 2 \\
\hline 49493 & 0 & 2 \\
\hline 49562 & 2 & 2 \\
\hline 49764 & 0 & 3 \\
\hline 49777 & 0 & 3 \\
\hline 49785 & 0 & 3 \\
\hline 49863 & 1 & 0 \\
\hline 49879 & 6 & 4 \\
\hline 49899 & 4 & 4 \\
\hline 50044 & 9 & 11 \\
\hline 50104 & 6 & 1 \\
\hline 50129 & 0 & 4 \\
\hline 50182 & 0 & 16 \\
\hline 50193 & 2 & 1 \\
\hline
\end{tabular}




\begin{tabular}{|c|c|c|}
\hline 50249 & 10 & 16 \\
\hline 50324 & 0 & 2 \\
\hline 50447 & 5 & 4 \\
\hline 50465 & 3 & 2 \\
\hline 50475 & 2 & 2 \\
\hline 50630 & 2 & 10 \\
\hline 50677 & 0 & 2 \\
\hline 50729 & 12 & 8 \\
\hline 50800 & 2 & 0 \\
\hline 50891 & 4 & 3 \\
\hline 50965 & 4 & 1 \\
\hline 50978 & 9 & 83 \\
\hline 50985 & 0 & 3 \\
\hline 51011 & 0 & 7 \\
\hline 51161 & 0 & 2 \\
\hline 51182 & 0 & 3 \\
\hline 51206 & 0 & 2 \\
\hline 51230 & 4 & 2 \\
\hline 51246 & 4 & 0 \\
\hline 51306 & 0 & 3 \\
\hline 51316 & 0 & 3 \\
\hline 51366 & 2 & 5 \\
\hline 51395 & 0 & 7 \\
\hline 51412 & 2 & 4 \\
\hline 51419 & 0 & 2 \\
\hline 51430 & 2 & 14 \\
\hline 51445 & 4 & 18 \\
\hline 51556 & 1 & 5 \\
\hline 51569 & 6 & 4 \\
\hline 51604 & 2 & 3 \\
\hline 51621 & 2 & 3 \\
\hline 51645 & 0 & 2 \\
\hline 51726 & 0 & 2 \\
\hline 51730 & 0 & 3 \\
\hline 51735 & 12 & 61 \\
\hline 51905 & 10 & 10 \\
\hline 51998 & 2 & 0 \\
\hline 52016 & 0 & 4 \\
\hline 52025 & 2 & 1 \\
\hline 52047 & 2 & 6 \\
\hline 52058 & 4 & 3 \\
\hline 52080 & 2 & 2 \\
\hline 52087 & 2 & 2 \\
\hline 52096 & 4 & 0 \\
\hline 52113 & 0 & 3 \\
\hline 52151 & 4 & 3 \\
\hline 52161 & 2 & 1 \\
\hline 52166 & 2 & 1 \\
\hline 52172 & 1 & 0 \\
\hline 52218 & 2 & 2 \\
\hline 52283 & 2 & 3 \\
\hline 52313 & 2 & 1 \\
\hline 52317 & 4 & 8 \\
\hline 52335 & 2 & 0 \\
\hline 52343 & 4 & 3 \\
\hline 52374 & 4 & 11 \\
\hline 52402 & 1 & 6 \\
\hline 52440 & 4 & 0 \\
\hline 52467 & 2 & 7 \\
\hline 52490 & 2 & 2 \\
\hline 52507 & 2 & 2 \\
\hline 52518 & 2 & 6 \\
\hline 52540 & 32 & 12 \\
\hline 52743 & 2 & 0 \\
\hline 52768 & 0 & 6 \\
\hline 52777 & 0 & 4 \\
\hline 52799 & 2 & 10 \\
\hline 52821 & 2 & 6 \\
\hline 52833 & 16 & 24 \\
\hline 52922 & 2 & 0 \\
\hline 52935 & 2 & 0 \\
\hline 52949 & 0 & 2 \\
\hline 52954 & 2 & 0 \\
\hline 52972 & 0 & 2 \\
\hline 53032 & 0 & 2 \\
\hline 53067 & 12 & 23 \\
\hline 53092 & 0 & 2 \\
\hline 53098 & 2 & 54 \\
\hline 53137 & 2 & 2 \\
\hline 53167 & 0 & 2 \\
\hline 53187 & 2 & 7 \\
\hline 53223 & 0 & 4 \\
\hline 53244 & 2 & 1 \\
\hline 53254 & 4 & 13 \\
\hline
\end{tabular}




\begin{tabular}{|c|c|c|}
\hline 53271 & 6 & 6 \\
\hline 53328 & 0 & 2 \\
\hline 53335 & 0 & 45 \\
\hline 53383 & 4 & 30 \\
\hline 53448 & 12 & 12 \\
\hline 53474 & 9 & 31 \\
\hline 53613 & 0 & 9 \\
\hline 53623 & 2 & 1 \\
\hline 53633 & 2 & 0 \\
\hline 53649 & 2 & 4 \\
\hline 53659 & 2 & 5 \\
\hline 53686 & 0 & 6 \\
\hline 53697 & 1 & 2 \\
\hline 53720 & 0 & \\
\hline 53732 & 0 & 4 \\
\hline 53738 & 2 & 1 \\
\hline 53756 & 4 & 7 \\
\hline 53774 & 2 & 5 \\
\hline 53850 & 0 & 4 \\
\hline 53901 & 0 & 4 \\
\hline 53927 & 1 & 5 \\
\hline 54001 & 6 & 17 \\
\hline 54189 & 2 & 2 \\
\hline 54197 & 3 & 4 \\
\hline 54211 & 6 & 15 \\
\hline 54236 & 4 & 12 \\
\hline 54427 & 0 & 3 \\
\hline 54495 & 2 & 13 \\
\hline 54534 & 2 & 2 \\
\hline 54570 & 0 & 2 \\
\hline 54575 & 2 & 0 \\
\hline 54616 & 2 & 0 \\
\hline 54626 & 0 & 2 \\
\hline 54631 & 2 & 2 \\
\hline 54637 & 0 & 5 \\
\hline 54686 & 0 & 2 \\
\hline 54695 & 2 & 2 \\
\hline 54747 & 2 & 0 \\
\hline 54768 & 0 & 5 \\
\hline 54791 & 0 & 3 \\
\hline 54826 & 2 & 3 \\
\hline 54843 & 2 & 1 \\
\hline 54849 & 0 & 6 \\
\hline 54897 & 2 & 2 \\
\hline 54928 & 10 & 40 \\
\hline 54980 & 2 & 0 \\
\hline 54999 & 0 & 2 \\
\hline 55021 & 2 & 2 \\
\hline 55035 & 2 & 0 \\
\hline 55060 & 2 & 3 \\
\hline 55103 & 0 & 2 \\
\hline 55120 & 4 & 4 \\
\hline 55129 & 2 & 2 \\
\hline 55154 & 2 & 0 \\
\hline 55174 & 2 & 3 \\
\hline 55190 & 2 & 0 \\
\hline 55205 & 0 & 2 \\
\hline 55257 & 0 & 4 \\
\hline 55277 & 2 & 0 \\
\hline 55282 & 2 & 1 \\
\hline 55298 & 2 & 0 \\
\hline 55307 & 2 & 2 \\
\hline 55315 & 4 & 4 \\
\hline 55424 & 2 & 1 \\
\hline 55455 & 2 & 2 \\
\hline 55469 & 0 & 2 \\
\hline 55486 & 2 & 4 \\
\hline 55608 & 0 & 6 \\
\hline 55666 & 0 & 2 \\
\hline 55681 & 2 & 5 \\
\hline 55729 & 0 & 18 \\
\hline 55753 & 0 & 3 \\
\hline 55797 & 2 & 1 \\
\hline 55811 & 0 & 7 \\
\hline 55821 & 0 & 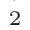 \\
\hline 55856 & 0 & 5 \\
\hline 55874 & 2 & 0 \\
\hline 55920 & 0 & 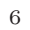 \\
\hline 55957 & 2 & 1 \\
\hline 55973 & 2 & \\
\hline 55979 & 0 & 2 \\
\hline 56019 & 0 & 3 \\
\hline 56047 & 0 & 3 \\
\hline 56053 & 0 & \\
\hline
\end{tabular}




\begin{tabular}{|c|c|}
\hline 56059 & 4 \\
\hline 56104 & \\
\hline 56112 & \\
\hline 56204 & \\
\hline 56219 & 4 \\
\hline 56235 & \\
\hline 56281 & 3 \\
\hline 56300 & \\
\hline 56317 & \\
\hline 56425 & \\
\hline 56542 & 2 \\
\hline 56634 & \\
\hline 56655 & \\
\hline 56672 & \\
\hline 56752 & \\
\hline 56784 & \\
\hline 56801 & \\
\hline 56808 & 2 \\
\hline 56815 & \\
\hline 56988 & ( \\
\hline 57196 & \\
\hline 57667 & \\
\hline 57701 & \\
\hline 57716 & 4 \\
\hline 57756 & 2 \\
\hline 57783 & \\
\hline 57829 & \\
\hline 57850 & \\
\hline 57863 & 2 \\
\hline 57868 & \\
\hline 57879 & 2 \\
\hline 57903 & 2 \\
\hline 63380 & 2 \\
\hline 63393 & c \\
\hline 63411 & \\
\hline 63737 & 2 \\
\hline 63748 & \\
\hline 64005 & ( \\
\hline 64153 & \\
\hline 64197 & 2 \\
\hline 64268 & \\
\hline 64356 & ( \\
\hline 64484 & ( \\
\hline 68906 & 2 \\
\hline 69000 & c \\
\hline 69322 & . \\
\hline 69572 & 2 \\
\hline 69593 & 2 \\
\hline 69645 & 0 \\
\hline 74650 & c \\
\hline 74924 & c \\
\hline 75217 & 2 \\
\hline 75304 & (c) \\
\hline 75553 & ( \\
\hline 75620 & ( \\
\hline 75632 & \\
\hline 81271 & ( \\
\hline 81296 & $\epsilon$ \\
\hline 81321 & 2 \\
\hline 81333 & 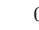 \\
\hline 81338 & ( \\
\hline 81342 & ( \\
\hline 81343 & 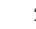 \\
\hline 81383 & . \\
\hline 81406 & \\
\hline 81442 & ( \\
\hline 81606 & 2 \\
\hline 81631 & 2 \\
\hline 81653 & 2 \\
\hline 81660 & \\
\hline 81665 & ( \\
\hline 81811 & \\
\hline 81901 & 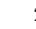 \\
\hline 81995 & \\
\hline 82061 & \\
\hline 82109 & \\
\hline 82199 & \\
\hline 82215 & \\
\hline 82282 & \\
\hline 82549 & \\
\hline 82649 & \\
\hline 82657 & \\
\hline 82754 & \\
\hline 82919 & 2 \\
\hline
\end{tabular}




$\begin{array}{lll}88697 & 1 & 2 \\ 88713 & 0 & 2 \\ 88723 & 0 & 6 \\ 88798 & 0 & 2 \\ 89000 & 4 & 0 \\ 89009 & 4 & 1 \\ 89124 & 0 & 3 \\ 89360 & 0 & 2 \\ 89942 & 0 & 2 \\ 90096 & 0 & 2 \\ 90123 & 2 & 0 \\ 90229 & 2 & 0 \\ 100920 & 6 & 1 \\ 100934 & 2 & 3 \\ 100950 & 4 & 6 \\ 101152 & 0 & 2 \\ 101447 & 0 & 3 \\ 101473 & 0 & 2 \\ 101489 & 2 & 0 \\ 101576 & 2 & 1 \\ 102114 & 0 & 2 \\ 102712 & 0 & 2 \\ 102860 & 2 & 0 \\ 103111 & 0 & 2 \\ 103243 & 2 & 0 \\ 103473 & 22 & 68 \\ 103481 & 3 & 3 \\ 103506 & 10 & 24 \\ 109993 & 0 & 2 \\ 110296 & 0 & 6 \\ 110921 & 2 & 0 \\ 110942 & 2 & 0 \\ 111331 & 2 & 1 \\ 111352 & 2 & 1 \\ 111430 & 2 & 1 \\ 195 & & 2\end{array}$

Table S3: List of the SCOP superfamily domains appearing in duplications and their relative population in the WGD and non-WGD sets of duplicates. 